\title{
DG-modules over de Rham DG-algebra
}

\author{
Sergey Rybakov
}

Received: 8 September 2014 / Accepted: 9 September 2014 / Published online: 16 December 2014 (C) Springer International Publishing AG 2014

\begin{abstract}
For a morphism of smooth schemes over a regular affine base we define functors of derived direct image and extraordinary inverse image on coderived categories of DG-modules over de Rham DG-algebras. Positselski proved that for a smooth algebraic variety $X$ over a field $k$ of characteristic zero the coderived category of DGmodules over $\Omega_{X / k}^{\bullet}$ is equivalent to the unbounded derived category of quasi-coherent right $\mathcal{D}_{X}$-modules. We prove that our functors correspond to the functors of the same name for $\mathcal{D}_{X}$-modules under Positselski equivalence.
\end{abstract}

Keywords de Rham complex $\cdot$ D-module $\cdot$ Coderived category

Mathematics Subject Classification $14 \mathrm{~F} 05 \cdot 14 \mathrm{~F} 10 \cdot 14 \mathrm{~F} 40$

The paper was refereed since November 2013 by CEJM but withdrawn due to imposition of publishing fees and resubmitted to EJM.

Supported in part by the Simons Foundation, by RFBR Grant No. 14-01-93108, and by AG Laboratory GU-HSE, RF government grant, ag. 11 11.G34.31.0023.

S. Rybakov ( $\square)$

Poncelet Laboratory (UMI 2615), Independent University of Moscow,

11 Bolshoy Vlasyevskiy Per., 119002 Moscow, Russia

e-mail: rybakov@mccme.ru; rybakov.sergey@gmail.com

S. Rybakov

Institute for Information Transmission Problems, Russian Academy of Sciences,

19 Bolshoy Karetny Per., 127994 Moscow, Russia

S. Rybakov

Laboratory of Algebraic Geometry, NRU HSE, 7 Vavilova Str.,

117312 Moscow, Russia 


\section{Introduction}

Let $X$ be a smooth algebraic variety over a field $k$ of characteristic zero. Its de Rham complex $\Omega_{X}=\Omega_{X / k}^{\bullet}$ is a sheaf of DG-algebras, and quasi-coherent sheaves of DGmodules over $\Omega_{X}$ (we call them $\Omega_{X}$-modules) form a DG-category, but the derived category of this category is usually not interesting. Recently Positselski defined the coderived category $\mathrm{D}^{\mathrm{co}}\left(\Omega_{X}\right.$-qcoh) of quasi-coherent $\Omega_{X}$-modules and proved that the unbounded derived category of quasi-coherent left $\mathcal{D}_{X}$-modules $\mathrm{D}\left(\mathcal{D}_{X}\right.$-qcoh $)$ and the coderived category $\mathrm{D}^{\mathrm{co}}\left(\Omega_{X}-\mathrm{q}\right.$ coh $)$ are equivalent [11]. This result is a generalization of Koszul duality for symmetric and exterior algebras. In this paper we construct functors of derived tensor product, direct image and extraordinary inverse image on $\Omega_{X}$-modules and prove that these functors correspond to the functors of the same name for $\mathcal{D}_{X}$-modules under Positselski equivalence (see Sect. 9). For coherent $\Omega_{X}$-modules we define the duality functor which correspond to duality on $\mathcal{D}_{X}$-modules. Before this, we prove some preliminary results which have obvious $\mathcal{D}_{X}$-module counterparts.

Some of these constructions are well-known for DG-categories of $\Omega$-modules (for example, [7] and [2, 7.2]), but as far as we know there was no attempt to write down these definitions for coderived categories of $\Omega$-modules. We feel it is important to do it.

\section{Quasi-coherent sheaves over $\Omega_{X}$}

2.1 DG-rings and DG-modules A $D G$-ring is a graded ring $R=\bigoplus_{i \in \mathbb{Z}} R^{i}$ endowed with an odd derivation $d: R \rightarrow R$ of degree 1 such that $d^{2}=0$. We write $R^{\text {gr }}$ when we view $R$ as a graded ring (or a DG-ring with zero differential). We denote by $|x|$ the degree of a homogeneous element $x \in R^{|x|}$. A DG-ring is called commutative if $x y=(-1)^{|x||y|} y x$ for any pair of homogeneous elements $x$ and $y$ of $R$. In what follows we assume that $R$ is a commutative DG-ring.

A (left) $D G$-module $M$ over $R$ is a graded $R$-module $M=\bigoplus_{i \in \mathbb{Z}} M^{i}$ endowed with differential $d_{M}: M \rightarrow M$ of degree 1 such that $d_{M}^{2}=0$, and for any pair of homogeneous elements $x \in R$ and $m \in M$ the following Leibniz rule holds:

$$
d_{M}(x m)=d x \cdot m+(-1)^{|x|} x d_{M}(m) .
$$

The module $M[j]=\bigoplus_{i \in \mathbb{Z}} M^{i+j}$ is called the shift of $M$ by an integer $j$. The differential on $M[j]$ is defined as $(-1)^{j} d_{M}$.

Let $M$ and $N$ be DG-modules over $R$. The DG-module of homomorphisms $\operatorname{Hom}_{R}(M, N)$ from $M$ to $N$ over $R$ is defined as follows. The component $\operatorname{Hom}_{R}^{i}(M, N)$ consists of all homogeneous maps $f: M \rightarrow N$ of degree $i$ such that $f(x m)=(-1)^{i|x|} x f(m)$ for homogeneous $x \in R$ and $m \in M$. Since $R$ is commutative, the formula $(x f)(m)=x f(m)$ defines an action of $R$ on $\operatorname{Hom}_{R}(M, N)$. The differential in the complex $\operatorname{Hom}_{R}(M, N)$ is given by the formula $d f(m)=$ $d_{N}(f(m))-(-1)^{|f|} f\left(d_{M}(m)\right)$. Clearly, $d^{2}(f)=0$, and for any composable morphisms $f$ and $g$ one has $d(f g)=d f \cdot g+(-1)^{|f|} f d g$. 
We define tensor product $D G$-module $M \otimes_{R} N$ of DG-modules $M$ and $N$ over $R$ as the tensor product $M^{\mathrm{gr}} \otimes_{R^{\mathrm{gr}}} N^{\mathrm{gr}}$ with the differential given by the formula $d(m \otimes n)=$ $d m \otimes n+(-1)^{|m|} m \otimes d n$.

2.2 DG-modules over the de Rham DG-algebra In this paper we always assume that all schemes are smooth, quasi-projective and connected over a regular connected affine base $S$. We denote by $\operatorname{dim} X$ the Krull dimension of $X$, and by $\Omega_{X}$ the sheaf of de Rham DG-algebras $\Omega_{X / S}^{\bullet}$. Note that $\Omega_{X}$ is a sheaf of commutative DG-algebras over $\mathcal{O}_{X}$. In what follows sheaves of DG-modules over $\Omega_{X}$ are assumed to be quasi-coherent over $\mathcal{O}_{X}$. We call them $\Omega_{X}$-modules for short. $\Omega_{X}$-modules form a DG-category $\Omega_{X}$-qcoh with shifts, cones and arbitrary direct sums. The complex of homomorphisms $\operatorname{Hom}_{\Omega_{X}}(M, N)$ from $M$ to $N$ over $\Omega_{X}$ is defined in a similar way as in the previous section. The component $\operatorname{Hom}_{\Omega_{X}}^{i}(M, N)$ consists of all homogeneous maps $f: M \rightarrow$ $N$ of degree $i$ such that over any affine open $U \subset X$ and for any $\omega \in \Omega_{U}$ and $m \in M(U)$ the following relation holds: $f(\omega m)=(-1)^{i|\omega|} \omega f(m)$. The formula for the differential is the same as before. Note that $\operatorname{Hom}_{\Omega_{X}}^{\bullet}(M, N)$ is a complex of $\mathcal{O}_{S}(S)$-modules.

An $\Omega_{X}$-module $M$ is called coherent if $M$ is coherent as an $\mathcal{O}_{X}$-module. In particular, there is a finite set of indexes $i$ such that the graded component $M^{i}$ is not zero. Coherent $\Omega_{X}$-modules form a DG-category $\Omega_{X}$-coh with shifts, cones and finite direct sums.

Let $M$ be a coherent $\Omega_{X}$-module. In this case we can define an $\Omega_{X}$-module $\mathcal{H}$ Hom $\Omega_{X}(M, N)$ of homomorphisms from $M$ to $N$ over $\Omega_{X}$. For any open $U \subset X$ the DG-module $\mathcal{H o m}_{\Omega_{X}}(M, N)(U)$ is the DG-module of homomorphisms $\operatorname{Hom}_{\Omega_{U}}(M(U)$, $N(U)$ ). Since $M$ is coherent, $\mathcal{H o m}_{\Omega_{X}}(M, N)$ is quasi-coherent.

Let $M$ and $N$ be $\Omega_{X}$-modules. One defines the tensor product $\Omega_{X}$-module $M \otimes_{\Omega_{X}} N$ as the associated sheaf to the presheaf $U \mapsto M(U) \otimes \Omega_{U} N(U)$.

We define a closed morphism $f$ as an element of $\operatorname{Hom}_{\Omega_{X}}^{0}(M, N)$ such that $d(f)=$ 0 . The $\Omega_{X}$-module $M$ is called contractible if the identity morphism is homotopic to zero, i.e. $\operatorname{Id}_{M}=d(h)$ for some $h \in \operatorname{Hom}_{\Omega_{X}}^{-1}(M, N)$. Let $f: M \rightarrow N$ be a closed morphism of $\Omega_{X}$-modules. The cone of $f$ is the following $\Omega_{X}$-module. As a graded module cone $(f)=M[1] \oplus N$, and the differential is given by the formula

$$
d_{\text {cone }(f)}(m, n)=\left(-d_{M}(m), f(m)+d_{N}(n)\right) .
$$

There is an exact sequence of $\Omega_{X}$-modules with closed differentials

$$
0 \rightarrow N \rightarrow \operatorname{cone}(f) \rightarrow M[1] \rightarrow 0 .
$$

We need the following generalization of the cone. Let $\cdots \rightarrow M_{i} \rightarrow M_{i-1} \rightarrow \cdots$ be a complex of $\Omega_{X}$-modules with closed differentials $d_{i}: M_{i} \rightarrow M_{i-1}$. Take the graded module $\operatorname{Tot}^{\oplus}\left(M_{\bullet}\right)=\bigoplus_{i} M_{i}[i]$ with differential

$$
d(m)=(-1)^{i} d_{M_{i}}(m)+d_{i}(m), \quad m \in M_{i} .
$$


The $\Omega_{X}$-module $\operatorname{Tot}^{\oplus}\left(M_{\bullet}\right)$ is called the total object of the complex $M_{\bullet}$ formed by (infinite) direct sums.

Let $D$ be a DG-category with shifts and cones. The homotopy category $\operatorname{Hot}(D)$ is the additive category with the same class of objects as $D$ and groups of morphisms given by $\operatorname{Hom}_{H o t}(D)(X, Y)=H^{0}\left(\operatorname{Hom}_{D}(X, Y)\right)$. The homotopy category $\operatorname{Hot}(D)$ is a triangulated category [3].

\section{Positselski results on coderived categories}

This section is an overview of some results on exotic derived categories due to Positselski. We present here a down to earth treatment of the subject [12]. The reader can find the results in full generality in the papers [9-11,13].

3.1 Fix an exact subcategory $E$ in the abelian category of quasi-coherent graded $\Omega_{X}^{\mathrm{gr}}$-modules. We say that $\Omega_{X}$-module is an $E$-module if its underlying graded module belongs to $E$. An $\Omega_{X}$-module is called absolutely acyclic with respect to $E$ if it belongs to the minimal thick subcategory of the homotopy category Hot $\left(\Omega_{X}\right.$-qcoh) containing total objects of exact triples of $E$-modules. We denote this subcategory by Acycl $_{E}^{\text {abs }}$. The quotient category of the homotopy category of $E$-modules by $\mathrm{Acycl}_{E}^{\text {abs }}$ is called the absolute derived category of E-modules.

For example, let $E$ be the subcategory of coherent $\Omega_{X}^{\mathrm{gr}}$-modules. Then $\mathrm{D}^{\mathrm{abs}}\left(\Omega_{X}\right.$-coh) $=\operatorname{Hot}\left(\Omega_{X}\right.$-coh $) / \operatorname{Acycl}_{\text {coh }}^{\text {abs }}$ is the absolute derived category of coherent $\Omega_{X}$-modules.

Suppose that $E$ is closed under arbitrary direct sums. An $\Omega_{X}$-module is called coacyclic with respect to $E$ if it belongs to the minimal thick subcategory of the homotopy category $\operatorname{Hot}\left(\Omega_{X}\right.$-qcoh) containing total objects of exact triples of $E$-modules and closed under arbitrary direct sums. We denote this subcategory by $\operatorname{Acycl}_{E}^{\text {co }}$. The quotient category of the homotopy category of $E$-modules by $\operatorname{Acycl}_{E}^{\text {co }}$ is called the coderived category of $E$-modules.

If $E$ is the category of quasi-coherent $\Omega_{X}^{\mathrm{gr}}$-modules, we call coacyclic $\Omega_{X}$-modules with respect to $E$ coacyclic, and denote the subcategory of coacyclic $\Omega_{X}$-modules by $\mathrm{Acycl}^{\mathrm{co}}$. Similarly, the category of absolutely acyclic $\Omega_{X}$-modules $\mathrm{Acycl}^{\text {abs }}$ is the category of absolutely acyclic $\Omega_{X}$-modules with respect to $E$. The category $\mathrm{D}^{\mathrm{co}}\left(\Omega_{X^{-}} \mathrm{qcoh}\right)=\operatorname{Hot}\left(\Omega_{X}\right.$-qcoh $) / \mathrm{Acycl}^{\mathrm{co}}$ is called the coderived category of $\Omega_{X}$-modules. We call two $\Omega_{X}$-modules co-isomorphic if they are isomorphic in $\mathrm{D}^{\mathrm{co}}\left(\Omega_{X}\right.$-qcoh). A curious example of co-isomorphism is given in Lemma 9.2.

A coherent absolutely acyclic $\Omega_{X}$-module is absolutely acyclic with respect to the category of coherent $\Omega_{X}^{\mathrm{gr}}$-modules, and the natural functor from $\mathrm{D}^{\mathrm{abs}}\left(\Omega_{X^{-}}\right.$coh) to $\mathrm{D}^{\mathrm{co}}\left(\Omega_{X}\right.$-qcoh $)$ is full and faithful $[11,3.11]$.

Lemma 3.2 (a) Let $M=\lim _{n \in \mathbb{N}} M_{n}$ be the limit of an inductive system of coacyclic $\Omega_{X}$-modules with closed morphisms. Then $M$ is coacyclic.

(b) Let $M_{0} \stackrel{d_{0}}{\rightarrow} M_{1} \rightarrow \cdots$ be an exact sequence of $\Omega_{X}$-modules with closed differentials. Then $M=\operatorname{ker} d_{0}$ and $\operatorname{Tot}^{\oplus} M_{\bullet}$ are co-isomorphic.

(c) Let $M=\bigcup_{n \in \mathbb{N}} M_{n}$ be an $\Omega_{X}$-module with an increasing filtration such that $M_{n+1} / M_{n}$ is coacyclic for any $n \in \mathbb{N}$. Then $M / M_{0}$ is coacyclic. 
Proof The first assertion follows from definitions since $M=\underline{\lim }_{n \in \mathbb{N}} M_{n}$ is the cone of the injective map id $-d: \bigoplus_{n \in \mathbb{N}} M_{n} \rightarrow \bigoplus_{n \in \mathbb{N}} M_{n}$. To prove the second assertion note that the total object of the sequence $0 \rightarrow M \rightarrow M_{0} \rightarrow \cdots \rightarrow M_{n} \rightarrow \operatorname{ker} d_{n+1} \rightarrow 0$ is absolutely acyclic. Hence the direct limit $N$ of such total objects is coacyclic, and we have an exact sequence of $\Omega_{X}$-modules

$$
0 \rightarrow \operatorname{Tot}^{\oplus} M_{\bullet} \rightarrow N \rightarrow M[-1] \rightarrow 0 .
$$

The third assertion follows from the fact that $M / M_{0}=\underline{\lim }_{n \in \mathbb{N}} M_{n} / M_{0}$.

3.3 An $\Omega_{X}$-module $I$ is called injective if $I$ is injective as a graded $\Omega_{X}^{\mathrm{gr}}$-module. By [13, Theorem A3], $I$ is injective if and only if the restriction of $I$ to any open subscheme $U$ is an injective $\Omega_{U}$-module. An $\Omega_{X}$-module $F$ is called flat if $F$ is flat as a graded $\Omega_{X}^{\mathrm{gr}}$-module. Let $M$ be an $\Omega_{X}$-module. We would like to find an injective $\Omega_{X}$-module $I$ and a flat $\Omega_{X}$-module $F$ which are co-isomorphic to $M$. As a first approximation to $I$ and $F$ we construct an injective $\Omega_{X}$-module $I_{0}$ with an injection $M \rightarrow I_{0}$ and a locally free (over $\Omega_{X}^{\mathrm{gr}}$ ) $\Omega_{X}$-module $P_{0}$ and a surjection $P_{0} \rightarrow M$.

There is an injective morphism of graded $\mathcal{O}_{X}$-modules $M \rightarrow J$ to an injective quasicoherent graded $\mathcal{O}_{X}$-module $J$ and a surjective $\mathcal{O}_{X}$-morphism $Q \rightarrow M$ from a locally free graded $\mathcal{O}_{X}$-module $Q$. We are going to construct functors $\mathrm{DG}^{+}$and $\mathrm{DG}^{-}$from graded $\mathcal{O}_{X}$-modules to $\Omega_{X}$-modules such that $I_{0}=\mathrm{DG}^{-}(J)$, and $P_{0}=\mathrm{DG}^{+}(Q)$.

3.3.1 Let $J$ be a quasi-coherent $\mathcal{O}_{X}$-module. The module $\mathrm{DG}^{-}(J)$ is cofreely generated by $J$ over $\Omega_{X}$. Namely, take a coinduced module $J^{\prime}=\mathcal{H} m_{\mathcal{O}_{X}}\left(\Omega_{X}^{\mathrm{gr}}, J\right)$. It is a graded $\Omega_{X}^{\mathrm{gr}}$-module such that for any $\Omega_{X}^{\mathrm{gr}}$-module $N$ there is a natural isomorphism

$$
\Phi: \mathcal{H o m}_{\mathcal{O}_{X}}(N, J) \rightarrow \mathcal{H o m}_{\Omega_{X}^{\mathrm{gr}}}\left(N, J^{\prime}\right) .
$$

The graded sheaf of abelian groups $\mathrm{DG}^{-}(J)$ is defined as follows. For an open $U \subset X$ the group $\mathrm{DG}^{-}(J)(U)$ consists of all formal expressions of the form $d^{-1} x+y$, where $x, y \in J^{\prime}(U)$, and $\operatorname{deg} d^{-1} x=\operatorname{deg} x-1$. The differential and the action of $\Omega_{U}$ on $\mathrm{DG}^{-}(J)(U)$ is given by the formulas: $d\left(d^{-1} x+y\right)=x$ and $\omega\left(d^{-1} x+y\right)=$ $\omega y+d^{-1}(d(\omega) y)+(-1)^{|\omega|} d^{-1}(\omega x)$ for $\omega \in \Omega_{U}$.

For any $\Omega_{X}$-module $M$ there is a bijective correspondence between morphisms of graded $\mathcal{O}_{X}$-modules $f: M \rightarrow J$ and closed morphisms of $\Omega_{X}$-modules $g: M \rightarrow \mathrm{DG}^{-}(J)$ which is described by the formula $g(m)=d^{-1}(\Phi(f)(d m))+$ $\Phi(f)(m)$. The module $\mathrm{DG}^{-}(J)$ is contractible. If $J$ is an injective $\mathcal{O}_{X}$-module, then $\mathrm{DG}^{-}(J) \cong J^{\prime} \oplus J^{\prime}[-1]$ as a graded $\Omega_{X}^{\mathrm{gr}}$-module.

3.3.2 The module $\mathrm{DG}^{+}(Q)$ is constructed in a similar way. As a graded sheaf of abelian groups

$$
\mathrm{DG}^{+}(Q)(U)=\left\{x+d y: x, y \in\left(Q \otimes \mathcal{O}_{X} \Omega_{X}\right)(U)\right\},
$$

where $\operatorname{deg} d y=\operatorname{deg} y+1$. The differential and the action of $\Omega_{U}$ on $\mathrm{DG}^{+}(Q)(U)$ is given by the formulas: $d(x+d y)=d x$ and $\omega(x+d y)=\omega x-(-1)^{|\omega|}(d \omega y)+$ $(-1)^{|\omega|} d(\omega y)$ for $\omega \in \Omega_{U}$. 
As before, there is a bijective correspondence between morphisms of graded $\mathcal{O}_{X^{-}}$ modules $f: Q \rightarrow M$ and closed morphisms of $\Omega_{X}$-modules $g: \mathrm{DG}^{+}(Q) \rightarrow M$, and $\mathrm{DG}^{+}(Q)$ is contractible. If $Q$ is locally free over $\mathcal{O}_{X}$, then $\mathrm{DG}^{+}(Q)$ is locally free as a graded $\Omega_{X}^{\mathrm{gr}}$-module.

3.4 Injective resolutions Denote the category of injective $\Omega_{X}$-modules by $\Omega_{X}-$ qcoh $_{\text {inj }}$.

Theorem The coderived category $\mathrm{D}^{\mathrm{co}}\left(\Omega_{X}\right.$-qcoh) is equivalent to the homotopy category $\operatorname{Hot}\left(\Omega_{X}-\mathrm{qcoh}_{\mathrm{inj}}\right)$.

Proof Let $M$ be an $\Omega_{X}$-module. Construct an injective resolution $I$ of $M$ as follows. By 3.3, we have an inclusion $M \rightarrow I_{0}$, where $I_{0}$ is injective. A common argument gives an exact complex $I_{\bullet}=\left(I_{0} \rightarrow I_{1} \rightarrow \cdots\right)$ of injective $\Omega_{X}$-modules with closed differentials. By Lemma $3.2(\mathrm{a}), I=\operatorname{Tot}^{\oplus} I_{\bullet}$ is co-isomorphic to $M$. Since $\Omega_{X}^{\mathrm{gr}}$ is Noetherian, the direct sum of injective modules is injective, thus $I$ is injective.

By [11, Theorem 3.5], for any coacyclic $\Omega_{X}$-module $L$ the complex $\operatorname{Hom}_{\Omega_{X}}(L, I)$ is acyclic. Thus $\operatorname{Hot}\left(\Omega_{X}-\mathrm{q} c \mathrm{~h}_{\mathrm{inj}}\right)$ and coacyclic $\Omega_{X}$-modules form a semiorthogonal decomposition of Hot $\left(\Omega_{X}-\right.$ qcoh $)$. By [11, Lemma 1.3], this proves the theorem.

3.5 Thick $\Omega_{X}$-modules We call an $\Omega_{X}$-module $A$ (co)induced if $A$ is (co)induced as an $\Omega_{X}^{\mathrm{gr}}$-module, i.e., there exists an $\mathcal{O}_{X}$-module $L$ such that $A^{\mathrm{gr}} \cong L \otimes_{\mathcal{O}_{X}} \Omega_{X}^{\mathrm{gr}}$ (or $\left.A^{\mathrm{gr}} \cong \mathcal{H o m}_{\mathcal{O}_{X}}\left(\Omega_{X}^{\mathrm{gr}}, L\right)\right)$. Note that any induced $\Omega_{X}^{\mathrm{gr}}$-module is coinduced and vice versa.

Theorem Let A be a quasi-coherent $\Omega_{X}^{\mathrm{gr}}$-module. The following conditions are equivalent:

(a) $\mathcal{T}_{\Omega_{X}}^{i} \mathrm{gr}\left(\mathcal{O}_{X}, A\right)=0$ for any $i>0$;

(b) $\mathcal{T}_{\Omega^{\prime}}^{i}{ }_{\Omega_{X}^{\mathrm{gr}}}^{\mathrm{gr}}(F, A)=0$ for any $i>0$, and for any $\mathcal{O}_{X}$-flat $\Omega_{X}^{\mathrm{gr}}$-module $F$;

(c) $\operatorname{Ext}_{\Omega_{X}^{i}}^{\mathrm{gr}}(A, J)=0$ for any $i>0$, and for any $\mathcal{O}_{X}$-injective $\Omega_{X}^{\mathrm{gr}}$-module $J$ such that $\Omega_{X}^{1} J=0$;

(d) $\operatorname{Ext}_{\Omega_{X}^{\mathrm{gr}}}^{i}(A, I)=0$ for any $i>0$, and for any $\mathcal{O}_{X}$-injective $\Omega_{X}^{\mathrm{gr}}$-module $I$;

(e) $\mathcal{E} x t_{\Omega_{X}^{i}}^{i}\left(\mathcal{O}_{X}, A\right)=0$ for any $i>0$;

(f) $\operatorname{Ext}_{\Omega_{X}^{i}}^{\mathrm{gr}}(P, A)=0$ for any $i>0$, and for any $\Omega_{X}^{\mathrm{gr}}$-module $P$ which is locally projective over $\mathcal{O}_{X}$.

If $A$ is (co)induced, then (a)-(f) hold.

Proof Clearly, (b) $\Rightarrow$ (a), (d) $\Rightarrow$ (c), (f) $\Rightarrow($ e), and if $A$ is (co)induced, then (a)-(f) hold. (a) $\Leftrightarrow\left(\right.$ c). Take a locally free over $\Omega_{X}^{\text {gr }}$ resolution for $A$

$$
\cdots \rightarrow P_{n} \rightarrow \cdots \rightarrow P_{0} \rightarrow A \rightarrow 0
$$

The complex

$$
\cdots \rightarrow P_{n} \otimes_{\Omega_{X}^{\mathrm{gr}}} \mathcal{O}_{X} \rightarrow \cdots \rightarrow P_{0} \otimes_{\Omega_{X}^{\mathrm{gr}}} \mathcal{O}_{X} \rightarrow 0
$$


computes $\mathcal{T}_{0} r_{\Omega_{X}^{i}}^{\mathrm{gr}}\left(\mathcal{O}_{X}, A\right)$. Apply $\mathcal{H} \mathrm{m}_{\mathcal{O}_{X}}(-, J)$ to this complex. We obtain a complex which computes $\mathcal{E x t}_{\Omega_{X}^{\bullet}}^{\mathrm{gr}}(A, J)$. Since $J$ is an arbitrary $\mathcal{O}_{X}$-injective module, the groups $\mathcal{E} x t_{\Omega_{X}^{i}}^{\mathrm{gr}}(A, J)$ are trivial if and only if $\mathcal{T}_{o r}{ }_{\Omega_{X}^{\mathrm{gr}}}^{\mathrm{gr}}\left(\mathcal{O}_{X}, A\right)=0$.

$(\mathrm{d}) \Rightarrow(\mathrm{b})$. Let $J$ be an injective $\mathcal{O}_{X}$-module. The module $I=\mathcal{H o m}_{\mathcal{O}_{X}}(F, J)$ is $\mathcal{O}_{X^{-}}$ injective $\Omega_{X}^{\text {gr }}$-module. Apply $\mathcal{H o m}_{\Omega_{X}^{\mathrm{gr}}}(-, I)$ to the resolution $(*)$. If (d) holds, we get a resolution for $\mathcal{H o m}_{\mathcal{O}_{X}}\left(A \otimes_{\Omega_{X}^{\mathrm{gr}}} F, J\right)$. Since $J$ is an arbitrary injective $\mathcal{O}_{X}$-module, $\mathcal{T}_{0}{ }_{\Omega_{X}^{\mathrm{gr}}}^{\mathrm{gr}}(F, A)=0$ for any $i>0$.

(a) $\Rightarrow$ (f). First, we prove a lemma.

Lemma Assume (a). Then A has a finite left resolution over $\Omega_{X}^{\mathrm{gr}}$ which is locally (co)induced.

Proof Take the truncation of the resolution $(*)$

$$
0 \rightarrow Q \rightarrow P_{n} \rightarrow \cdots \rightarrow P_{0} \rightarrow A \rightarrow 0
$$

where $n=\operatorname{dim} X$. Then $Q$ is locally projective $\mathcal{O}_{X}$-module. Tensor $(* *)$ with $\mathcal{O}_{X}$ over $\Omega_{X}^{\mathrm{gr}}$. By condition (a), the module $L=Q \otimes_{\Omega_{X}^{\mathrm{gr}}} \mathcal{O}_{X}$ is also locally projective over $\mathcal{O}_{X}$, and $\mathcal{T}_{0}{ }_{\Omega_{X}^{\mathrm{gr}}}^{i}\left(\mathcal{O}_{X}, Q\right)=0$ for any $i>0$. We have the natural surjective morphism of $\Omega_{X}^{\mathrm{gr}}$-modules

$$
Q \rightarrow L \cong Q / \Omega_{X}^{1} Q
$$

This implies that locally there is a morphism of $\mathcal{O}_{X}$-modules $L \rightarrow Q$ which induces a surjective morphism of $\Omega_{X}^{\mathrm{gr}}$-modules

$$
\varphi: L \otimes_{\mathcal{O}_{X}} \Omega_{X}^{\mathrm{gr}} \rightarrow Q
$$

Let $K=\operatorname{ker} \varphi$. Clearly, $\mathcal{T}_{o r}^{i}{ }_{\Omega_{X}}^{i \mathrm{gr}}\left(\mathcal{O}_{X}, K\right)=0$ for any $i \geq 0$, and thus $K=0$. We proved that locally $Q$ is a (co)induced module.

By the lemma, the module $A$ has a resolution

$$
0 \rightarrow P_{n} \rightarrow \cdots \rightarrow P_{0} \rightarrow A \rightarrow 0
$$

such that for any $P_{k}$ the property (f) holds: $\mathcal{E}_{\Omega_{\Omega_{X}}^{\mathrm{gr}}}^{i}\left(P, P_{k}\right)=0$ for any $i>0$. It follows that (f) is true for $A$. To prove (e) $\Rightarrow$ (d) one uses almost the same argument involving right resolution of $A$ instead of the resolution (*).

We call an $\Omega_{X}^{\mathrm{gr}}$-module $A$ thick if conditions (a)-(f) hold for $A$. We call $\Omega_{X^{-}}$ module thick if it is thick as a graded $\Omega_{X}^{\mathrm{gr}}$-module. Positselski call such modules weakly relatively admissible. The following result can be proved using the argument given in the previous lemma. 
Corollary Let A be a thick $\Omega_{X}^{\mathrm{gr}}$-module. Suppose that $A$ is locally projective (flat or injective) over $\mathcal{O}_{X}$. Then $A$ is locally projective (respectively flat or injective) over $\Omega_{X}^{\mathrm{gr}}$.

\subsection{Thick resolutions}

Theorem The coderived category $\mathrm{D}^{\mathrm{co}}\left(\Omega_{X}\right.$-qcoh) is equivalent to the absolute derived category of $\mathrm{D}^{\mathrm{abs}}\left(\Omega_{X}\right.$-qcoh $\left.{ }_{\text {thick }}\right)$ of thick $\Omega_{X}$-modules.

Proof We prove that subcategory of absolutely acyclic thick $\Omega_{X}$-modules and the homotopy category of injective $\Omega_{X}$-modules form a semiorthogonal decomposition of the homotopy category of thick $\Omega_{X}$-modules. The theorem then follows from [11, Lemma 1.3] and Theorem 3.4.

Let $A$ be a thick $\Omega_{X}$-module. Find an $\mathcal{O}_{X}$-injection of $A$ into an injective $\mathcal{O}_{X^{-}}$ module $J_{0}$. By 3.3 we obtain an inclusion $A \rightarrow I_{0}=\mathrm{DG}^{-}\left(J_{0}\right)$ into a coinduced injective $\Omega_{X}$-module. In this way we construct first terms of the resolution for $A$ by coinduced injective $\Omega_{X}$-modules

$$
I_{0} \rightarrow I_{1} \rightarrow \cdots \rightarrow I_{n}
$$

where all morphisms are closed. Let $J$ be the cokernel of the map $I_{n-1} \rightarrow I_{n}$. Then $J$ is thick. Moreover, if $n \geq \operatorname{dim} X$, then $J$ is injective over $\mathcal{O}_{X}$. By Corollary 3.5, $J$ is an injective $\Omega_{X}$-module. It follows that

$$
I=\operatorname{Tot}^{\oplus}\left(I_{0} \rightarrow \cdots \rightarrow I_{n} \rightarrow J\right)
$$

is injective too. Moreover, the cokernel $L$ of the natural morphism $A \rightarrow I$ is an absolutely acyclic thick $\Omega_{X}$-module. By [11, Theorem 3.5], $\operatorname{Hom}_{\Omega_{X}}(L, I)$ is acyclic (see also Lemma 4.1).

Remark In fact, we proved that any $\Omega_{X}$-module $M$ has a coinduced resolution, i.e. there exists a coinduced $\Omega_{X}$-module $A$ and a morphism $M \rightarrow A$ with absolutely acyclic cone.

\subsection{Flat resolutions}

Theorem The coderived category $\mathrm{D}^{\mathrm{co}}\left(\Omega_{X}\right.$-qcoh $)$ is equivalent to the absolute derived category $\mathrm{D}^{\mathrm{abs}}\left(\Omega_{X}\right.$ - $\left.\mathrm{qcoh} \mathrm{fl}_{\mathrm{fl}}\right)$ of flat $\Omega_{X}$-modules.

Proof By Theorem 3.6, $\mathrm{D}^{\mathrm{co}}\left(\Omega_{X}\right.$-qcoh) is equivalent to $\mathrm{D}^{\mathrm{abs}}\left(\Omega_{X}-\mathrm{qcoh}_{\text {thick }}\right)$. Let us prove that the latter is equivalent to $\mathrm{D}^{\mathrm{abs}}\left(\Omega_{X}-\mathrm{qcoh}_{\mathrm{fl}}\right)$. Let $A$ be a thick $\Omega_{X}$-module. As before, using 3.3 find a finite exact complex

$$
0 \rightarrow P_{n} \rightarrow \cdots \rightarrow P_{0} \rightarrow A \rightarrow 0
$$

of $\Omega_{X}$-modules such that $P_{0}, \ldots, P_{n-1}$ are flat (and even locally free over $\Omega_{X}^{\mathrm{gr}}$ ) and differentials are closed. If $n$ is greater than dimension of $X$, the module $P_{n}$ is also flat 
by Corollary 3.5. It follows that $P=\operatorname{Tot}^{\oplus} P \bullet$ is flat and cone $(P \rightarrow A)$ is absolutely acyclic with respect to $\Omega_{X}$-qcoh thick $_{\text {. }}$.

We proved that the natural functor

$$
\mathrm{D}^{\mathrm{abs}}\left(\Omega_{X}-\mathrm{qcoh}_{\text {thick }}\right) \rightarrow \mathrm{D}^{\mathrm{abs}}\left(\Omega_{X}-\mathrm{qcoh}_{\mathrm{fl}}\right)
$$

is essentially surjective. We have to show that it is full and faithful. The key point is to prove that any object of $\Omega_{X}-\mathrm{qcoh}_{\mathrm{fl}}$ that is absolutely acyclic with respect to $\Omega_{X}-$ qcoh $_{\text {thick }}$ is absolutely acyclic with respect to $\Omega_{X}-\mathrm{qcoh}_{\mathrm{fl}}$. This is done in [9, Sect. 3.2].

3.8 Perfect resolutions We need a one more type of resolutions. Denote the category of $\mathcal{O}_{X}$-locally free coherent $\Omega_{X}$-modules by $\Omega_{X}$-perf. We call such $\Omega_{X}$-modules perfect. This is an exact category and we can define the absolute derived category $\mathrm{D}^{\mathrm{abs}}\left(\Omega_{X}\right.$-perf $)$.

Theorem The absolute derived category $\mathrm{D}^{\mathrm{abs}}\left(\Omega_{X}\right.$-coh) of coherent $\Omega_{X}$-modules is equivalent to the absolute derived category $\mathrm{D}^{\mathrm{abs}}\left(\Omega_{X}\right.$-perf) of perfect $\Omega_{X}$-modules.

Proof Let $M$ be a coherent $\Omega_{X}$-module. By 3.3, there is a finite exact complex $0 \rightarrow$ $P_{n} \rightarrow \cdots \rightarrow P_{0} \rightarrow M \rightarrow 0$ of $\Omega_{X}$-modules such that $P_{0}, \ldots, P_{n-1}$ are perfect (and even locally free over $\Omega_{X}^{\mathrm{gr}}$ ) and differentials are closed. If $n \geq \operatorname{dim} X$, the module $P_{n}$ is also perfect. It follows that $\operatorname{Tot}^{\oplus} P_{\bullet}$ is perfect and co-isomorphic to $M$. As before, one can prove that any object of $\Omega_{X}$-perf that is absolutely acyclic with respect to $\Omega_{X}$-coh is absolutely acyclic with respect to $\Omega_{X}$-perf (see [9, Sect. 3.2]).

Remark For any $\Omega_{X}$-module $M$ there is a flat resolution $F$ which is locally free over $\Omega_{X}^{\mathrm{gr}}$. Indeed, the same construction as before gives a resolution $P$ of $M$ which is locally free over $\mathcal{O}_{X}$, and the coinduced resolution of $P$ is locally free over $\Omega_{X}^{\mathrm{gr}}$.

\section{Inverse and direct images}

Let $f: X \rightarrow Y$ be a morphism of smooth schemes over $S$. Denote by $f^{\bullet}$ and $f_{\bullet}$ the inverse and direct image of sheaves of abelian groups associated to $f$. We have a morphism of sheaves of DG-algebras on $X: f^{\bullet} \Omega_{Y} \rightarrow \Omega_{X}$. This gives a morphism of DG-ringed spaces $\left(X, \Omega_{X}\right) \rightarrow\left(Y, \Omega_{Y}\right)$, and allows one to define inverse and direct image functors on coderived categories of $\Omega$-modules.

4.1 Derived Hom and tensor product First we define functors of derived Hom and (shifted) tensor product:

$$
\begin{array}{r}
R \mathcal{H}{ }_{\Omega_{X}}: \mathrm{D}^{\mathrm{abs}}\left(\Omega_{X}-\mathrm{coh}\right) \times \mathrm{D}^{\mathrm{co}}\left(\Omega_{X}-\mathrm{qcoh}\right) \rightarrow \mathrm{D}^{\mathrm{co}}\left(\Omega_{X} \text {-qcoh }\right), \\
\otimes_{\Omega_{X}}^{D}: \mathrm{D}^{\mathrm{co}}\left(\Omega_{X} \text {-qcoh }\right) \times \mathrm{D}^{\mathrm{co}}\left(\Omega_{X} \text {-qcoh }\right) \rightarrow \mathrm{D}^{\mathrm{co}}\left(\Omega_{X}-\mathrm{qcoh}\right) .
\end{array}
$$

Lemma (a) For any absolutely acyclic coherent $\Omega_{X}$-module $M$ and injective $\Omega_{X^{-}}$ module I the $\Omega_{X}$-module $\mathcal{H o m}_{\Omega_{X}}(M, I)$ is absolutely acyclic. 
(b) For any coherent $\Omega_{X}$-module $M$ and coacyclic injective $\Omega_{X}$-module I the $\Omega_{X}$ module $\operatorname{Hom}_{\Omega_{X}}(M, I)$ is coacyclic.

(c) For any coacyclic $\Omega_{X}$-module $M$ and flat $\Omega_{X}$-module $F$ the $\Omega_{X}$-module $M \otimes_{\Omega_{X}} F$ is coacyclic.

(d) Let $F$ be a flat absolutely acyclic (with respect to the additive category of flat $\Omega_{X}$ modules) $\Omega_{X}$-module. Then for any $\Omega_{X}$-module $M$ the $\Omega_{X}$-module $M \otimes_{\Omega_{X}} F$ is coacyclic.

Proof The proof of (a) is almost the same as [11, Theorem 3.5]. Indeed, the functor $\mathcal{H o m}_{\Omega_{X}}(-, I)$ respects shifts, cones and direct sums. Thus it is enough to check (a) when $M$ is the total object of an exact triple of $\Omega_{X}$-modules. Clearly, $\mathcal{H}_{\Omega_{X}}(M, I)$ is the the total object of an exact triple of $\Omega_{X}$-modules. Similarly, if $I$ is co-acyclic, then $\operatorname{Hom}_{\Omega_{X}}(I, I)$ is acyclic, and $I$ is contractible. It follows that $\mathcal{H o m}_{\Omega_{X}}(M, I)$ is coacyclic. This proves (b).

The functor $-\otimes_{\Omega_{X}} F$ respects shifts, cones and arbitrary direct sums. We have to check (c) when $M$ is the total object of an exact triple of $\Omega_{X}$-modules and (d) when $F$ is the total object of an exact triple of flat $\Omega_{X}$-modules. Both times, $M \otimes_{\Omega_{X}} F$ is the the total object of an exact triple of $\Omega_{X}$-modules.

4.1.1 We are ready to define the functor $R \mathcal{H} \operatorname{Hom}_{\Omega_{X}}$. Take the restriction of the functor

$$
\mathcal{H} \operatorname{Hom}_{\Omega_{X}}: \operatorname{Hot}\left(\Omega_{X}-\operatorname{coh}\right) \times \operatorname{Hot}\left(\Omega_{X}-\mathrm{qcoh}\right) \rightarrow \operatorname{Hot}\left(\Omega_{X}-\mathrm{qcoh}\right)
$$

to the subcategory $\operatorname{Hot}\left(\Omega_{X}-\operatorname{coh}\right) \times \operatorname{Hot}\left(\Omega_{X}-\mathrm{qcoh}_{\mathrm{inj}}\right)$. By Lemma 4.1 (a), this restriction factors through the functor

$$
\mathrm{D}^{\mathrm{abs}}\left(\Omega_{X}-\mathrm{coh}\right) \times \operatorname{Hot}\left(\Omega_{X}-\mathrm{qcoh} \text { inj }\right) \rightarrow \mathrm{D}^{\mathrm{co}}\left(\Omega_{X}-\mathrm{qcoh}\right)
$$

By Theorem 3.4, Hot $\left(\Omega_{X}-\mathrm{qcoh}\right.$ inj $)$ is equivalent to $\mathrm{D}^{\mathrm{co}}\left(\Omega_{X}\right.$-qcoh). Combining this equivalence with the functor $(* * *)$ we get the functor $R \mathcal{H}{ }_{0} \Omega_{\Omega_{X}}$.

4.1.2 Let us define the derived tensor product. Let $n$ be the relative dimension of $X$ over $S$. By Lemma 4.1 (c)\&(d), the shifted tensor product

$$
\otimes_{\Omega_{X}}[2 n]: \operatorname{Hot}\left(\Omega_{X}-\mathrm{qcoh}\right) \times \operatorname{Hot}\left(\Omega_{X}-\mathrm{q} \operatorname{coh} \mathrm{fl}_{1}\right) \rightarrow \operatorname{Hot}\left(\Omega_{X}-\mathrm{qcoh}\right)
$$

factors through

$$
\mathrm{D}^{\mathrm{co}}\left(\Omega_{X}-\mathrm{qcoh}\right) \times \mathrm{D}^{\mathrm{abs}}\left(\Omega_{X}-\mathrm{qcoh}_{\mathrm{fl}}\right) \rightarrow \mathrm{D}^{\mathrm{co}}\left(\Omega_{X}-\mathrm{qcoh}\right) .
$$

By Theorem 3.7, $\mathrm{D}^{\mathrm{abs}}\left(\Omega_{X}-\mathrm{qcoh}_{\mathrm{fl}}\right)$ is equivalent to $\mathrm{D}^{\mathrm{co}}\left(\Omega_{X}\right.$-qcoh $)$, and we are done. The tensor product can be obtained in two equivalent ways

$$
\begin{aligned}
\mathrm{D}^{\mathrm{co}}\left(\Omega_{X}-\mathrm{qcoh}\right) \times \mathrm{D}^{\mathrm{co}}\left(\Omega_{X}-\mathrm{qcoh}\right) & \rightarrow \mathrm{D}^{\mathrm{abs}}\left(\Omega_{X}-\mathrm{qcoh}_{\mathrm{fl}}\right) \times \mathrm{D}^{\mathrm{co}}\left(\Omega_{X}-\mathrm{qcoh}\right) \\
& \stackrel{\Omega_{\Omega_{X}}[2 n]}{\longrightarrow} \mathrm{D}^{\mathrm{co}}\left(\Omega_{X}-\mathrm{qcoh}\right),
\end{aligned}
$$


where the first arrow comes from the equivalence 3.7, or

$$
\begin{aligned}
\mathrm{D}^{\mathrm{co}}\left(\Omega_{X}-\mathrm{qcoh}\right) \times \mathrm{D}^{\mathrm{co}}\left(\Omega_{X}-\mathrm{qcoh}\right) & \rightarrow \mathrm{D}^{\mathrm{co}}\left(\Omega_{X}-\mathrm{qcoh}\right) \times \mathrm{D}^{\mathrm{abs}}\left(\Omega_{X}-\mathrm{qcoh}_{\mathrm{fl}}\right) \\
& \stackrel{\Omega_{\Omega_{X}}[2 n]}{\longrightarrow} \mathrm{D}^{\mathrm{co}}\left(\Omega_{X}-\mathrm{qcoh}\right) .
\end{aligned}
$$

These definitions of tensor product are equivalent since both functors factors through $\mathrm{D}^{\mathrm{abs}}\left(\Omega_{X}-\mathrm{qcoh} \mathrm{fl}_{\mathrm{f}}\right) \times \mathrm{D}^{\mathrm{abs}}\left(\Omega_{X}-\mathrm{qcoh}_{\mathrm{fl}}\right)$.

Theorem 4.2 Let $P$ be a perfect $\Omega_{X}$-module, and A be a thick $\Omega_{X}$-module. Then

(a) $R \mathcal{H o m}_{\Omega_{X}}(P, A) \cong \operatorname{Hom}_{\Omega_{X}}(P, A)$, and

(b) $P \otimes_{\Omega_{X}}^{D} A \cong P \otimes_{\Omega_{X}} A[2 n]$

in $\mathrm{D}^{\mathrm{co}}\left(\Omega_{X}-\mathrm{qcoh}\right)$.

Proof We prove (a) and leave (b) to the reader. Take an injective resolution of $A$ from the proof of Theorem 3.6:

$$
I=\operatorname{Tot}^{\oplus}\left(I_{0} \rightarrow \cdots \rightarrow I_{n} \rightarrow J\right)
$$

We know that exact sequence $I_{\bullet}=\left(I_{0} \rightarrow \cdots \rightarrow I_{n} \rightarrow J\right)$ gives an injective resolution of $A$ over $\Omega_{X}^{\mathrm{gr}}$. Since $P$ is perfect, the complex of $\Omega_{X}^{\mathrm{gr}}$-modules $\mathcal{H o m}_{\Omega_{X}^{\mathrm{gr}}}\left(P^{\mathrm{gr}}, I_{\bullet}^{\mathrm{gr}}\right)$ has homology at zero term only. It follows that $\mathcal{H o m}_{\Omega_{X}}(P, I)$ is co-isomorphic to $\mathcal{H o m}_{\Omega_{X}}(P, A)$.

4.3 Direct image In this section we define the functor of direct image

$$
f_{*}: \mathrm{D}^{\mathrm{co}}\left(\Omega_{X}-\mathrm{qcoh}\right) \rightarrow \mathrm{D}^{\mathrm{co}}\left(\Omega_{Y}-\mathrm{qcoh}\right)
$$

For any injective $\Omega_{X}$ module $I$ the direct image $f_{\bullet}(I)$ is an $\Omega_{Y}$-module since $f$ is a morphism of DG-ringed spaces $\left(X, \Omega_{X}\right) \rightarrow\left(Y, \Omega_{Y}\right)$. This gives the functor

$$
f_{\circ}: \operatorname{Hot}\left(\Omega_{X}-\mathrm{qcoh}_{\mathrm{inj}}\right) \rightarrow \mathrm{D}^{\mathrm{co}}\left(\Omega_{Y}-\mathrm{qcoh}\right) .
$$

Any $\Omega_{X}$-module has an injective resolution, and we define $f_{*}$ as a composition of $f_{\circ}$ with the equivalence 3.4. It is well known that for an injective sheaf $I$ the sheaf $f_{\bullet}(I)$ is flabby, and $f_{\bullet}$ is exact on flabby sheaves. Thus, for a pair of composable maps $f$ and $g$ we have $(f \circ g)_{*} \cong f_{*} \circ g_{*}$.

By definition, $\Omega_{S} \cong \mathcal{O}_{S}$, and $\operatorname{Hom}_{\Omega_{X}}(M, N)$ is a complex of $\mathcal{O}_{S}(S)$-modules. Since $\mathcal{O}_{S}$ has finite homological dimension and affine,

$$
\mathrm{D}^{\mathrm{co}}\left(\Omega_{S}-\mathrm{qcoh}\right) \cong \mathrm{D}\left(\mathcal{O}_{S}(S)-\bmod \right)
$$

is an unbounded derived category of $\mathcal{O}_{S}(S)$-modules [10, Remark 2.1]. 
Proposition Let $f$ be the morphism from $X$ to $S, N \in \mathrm{D}^{\mathrm{co}}\left(\Omega_{X}\right.$-qcoh) and $M \in$ $\mathrm{D}^{\mathrm{abs}}\left(\Omega_{X}\right.$-coh). Then there is a canonical isomorphism in $\mathrm{D}\left(\mathcal{O}_{S}(S)\right.$-mod)

$$
\Gamma\left(S, f_{*} R \mathcal{H o m}_{\Omega_{X}}(M, N)\right) \rightarrow \operatorname{Hom}_{\Omega_{X}}(M, I),
$$

where $I$ is an injective resolution of $N$. In particular, the space of homomorphisms $\operatorname{Hom}_{D^{\mathrm{co}}\left(\Omega_{X} \text {-qcoh }\right)}(M, N)$ is isomorphic to $H^{0}\left(S, f_{*} R \mathcal{H o m}_{\Omega_{X}}(M, N)\right)$.

Proof The $\Omega_{X}$-module $\mathcal{H o m}_{\Omega_{X}}(M, I)$ is flabby. Thus

$$
\Gamma\left(S, f_{*} R \mathcal{H o m}_{\Omega_{X}}(M, N)\right) \cong \Gamma\left(S, f_{\bullet} \mathcal{H}_{0} m_{\Omega_{X}}(M, I)\right) \cong \operatorname{Hom}_{\Omega_{X}}(M, I) .
$$

4.4 Extraordinary inverse image Now, we define two inverse image functors:

$$
f^{+}, f^{!}: \mathrm{D}^{\mathrm{co}}\left(\Omega_{Y^{-}} \mathrm{qcoh}\right) \rightarrow \mathrm{D}^{\mathrm{co}}\left(\Omega_{X^{-}} \mathrm{qcoh}\right) .
$$

By Theorem 3.7, it is enough to define the functors on $\mathrm{D}^{\mathrm{co}}\left(\Omega_{Y}-\mathrm{qcoh}_{\mathrm{fl}}\right)$. We do it as follows:

$$
f^{+}(F)=f^{\bullet}(F) \otimes_{f} \Omega_{Y} \Omega_{X},
$$

where $F$ is flat. The extraordinary inverse image is defined as $f^{!}(M)=f^{+}(M)$ [2( $\operatorname{dim} X-\operatorname{dim} Y)]$. Clearly, for a pair of composable maps $f$ and $g$ we have $(f \circ g)^{!} \cong$ $g ! \circ f^{!}$.

Lemma Suppose that $f: X \rightarrow Y$ is smooth. Then $\Omega_{X}$ is flat over $f^{\bullet} \Omega_{Y}$.

Proof It follows from the smoothness of $f$ that $\Omega_{X}^{\mathrm{gr}}$ is locally free over $f^{\bullet}\left(\Omega_{Y}^{\mathrm{gr}}\right)$ $\otimes_{f} \bullet \mathcal{O}_{Y} \mathcal{O}_{X}$ and $\mathcal{O}_{X}$ is flat over $f^{\bullet} \mathcal{O}_{Y}$. Thus $\Omega_{X}^{\mathrm{gr}}$ is flat over $f^{\bullet}\left(\Omega_{Y}^{\mathrm{gr}}\right)$.

Corollary Suppose that $f: X \rightarrow Y$ is smooth, and $M$ is an $\Omega_{Y}$-module. Then

(a) $f^{+}(M) \cong f^{\bullet}(M) \otimes_{f} \bullet \Omega_{Y} \Omega_{X}$.

(b) The extraordinary inverse image of a coherent $\Omega_{Y}$-module is coherent.

(c) If $f$ is an open embedding, then $f^{!}(M) \cong f^{+}(M) \cong f^{\bullet}(M) \otimes_{f} \bullet \mathcal{O}_{Y} \mathcal{O}_{X}$.

Theorem 4.5 Suppose that $f$ is smooth. Then functors $f_{*}$ and $f^{+}$are adjoint. Let $N \in \mathrm{D}^{\mathrm{co}}\left(\Omega_{X^{-}} \mathrm{qcoh}\right)$ and let $M \in \mathrm{D}^{\mathrm{abs}}\left(\Omega_{X^{-}}-\mathrm{coh}\right)$. Then

$$
R \mathcal{H o m}_{\Omega_{Y}}\left(M, f_{*} N\right) \cong f_{*} R \mathcal{H o m}_{\Omega_{X}}\left(f^{+} M, N\right)
$$

Proof We prove the second assertion. The same argument proves that the functors are adjoint. Take an injective resolution $I$ for $N$. Since the functor $f^{\bullet}$ is exact, and functors $f_{\bullet}$ and $f^{\bullet}$ are adjoint, the module $f_{\bullet}(I)$ is injective. Moreover, homomorphism sheaf to an injective sheaf is flabby. Thus we have to prove that

$$
\mathcal{H o m}_{\Omega_{Y}}\left(M, f_{\bullet} I\right) \cong f_{\bullet} \mathcal{H o m}_{\Omega_{X}}\left(f^{\bullet} M \otimes_{f} \bullet \Omega_{Y} \Omega_{X}, I\right) .
$$

And this can be shown in the usual way. 
Theorem 4.6 (projection formula) Let $f: X \rightarrow Y$ be a morphism of smooth schemes over $S$. Let $M$ be an $\Omega_{X}$-module and let $N$ be an $\Omega_{Y}$-module. Then $N \otimes_{\Omega_{X}}^{D} f_{*} M \cong$ $f_{*}\left(f^{!} N \otimes_{\Omega_{Y}}^{D} M\right)$ in $\mathrm{D}^{\mathrm{co}}\left(\Omega_{Y}-\mathrm{qcoh}\right)$.

Proof Let $I$ be an injective resolution of $M$, and $F$ be a flat resolution of $N$, which is locally free over $\Omega_{Y}^{\mathrm{gr}}$. We have the natural morphism

$$
F \otimes_{\Omega_{Y}} f_{*} I \rightarrow f_{\bullet}\left(f^{+} F \otimes_{\Omega_{X}} I\right)
$$

Take the composition $\Phi$ of this morphism with

$$
f_{\bullet}\left(f^{+} F \otimes_{\Omega_{X}} I\right) \rightarrow f_{*}\left(f^{+} F \otimes_{\Omega_{X}} I\right) .
$$

We claim that $\Phi$ is an isomorphism. It is enough to check the claim for graded modules. Clearly, $\Phi$ is an isomorphism when $F=\Omega_{Y}^{\mathrm{gr}}$. Thus the claim holds for any locally free $\Omega_{Y}^{\mathrm{gr}}$-module. The projection formula now follows from the definition of the (shifted) derived tensor product and of the extraordinary inverse image.

4.7 Extraordinary inverse image for a closed embedding Let $i: Z \rightarrow X$ be a closed embedding of smooth schemes over $S$. In this case $\Omega_{Z}$ is coherent over $\Omega_{X}$, and we can give the second definition of the extraordinary inverse image

$$
i^{b}(M)=\mathcal{H o m}_{i} \bullet \Omega_{X}\left(\Omega_{Z}, i^{\bullet}(M)\right) .
$$

We define the derived functor $R i^{b}: \mathrm{D}^{\mathrm{co}}\left(\Omega_{Y}\right.$-qcoh $) \rightarrow \mathrm{D}^{\mathrm{co}}\left(\Omega_{X}\right.$-qcoh $)$ using the equivalence 3.4 .

Let $I$ be an injective $\Omega_{X}$-module. Since functors $i_{\bullet}, \mathcal{H o m}_{i} \bullet \Omega_{X}^{\mathrm{gr}}\left(\Omega_{Z}^{\mathrm{gr}}, i^{\bullet}(-)\right)$ are adjoint, and $i_{\bullet}$ is exact, the $\Omega_{X}$-module $i^{b}(I)$ is injective. It follows that for any pair of composable closed embeddings $f$ and $g$ we have $(f \circ g)^{b} \cong g^{b} \circ f^{b}$. For the same reason, we have

Theorem 4.7 The functors $i_{*}$ and $R i^{b}$ are adjoint. Let $N \in \mathrm{D}^{\mathrm{co}}\left(\Omega_{X}\right.$-qcoh) and let $M \in \mathrm{D}^{\mathrm{abs}}\left(\Omega_{X}-\mathrm{coh}\right)$. Then

$$
i_{*} R \mathcal{H o m}_{\Omega_{Z}}\left(M, R i^{b}(N)\right) \cong R \mathcal{H o m}_{\Omega_{X}}\left(i_{*} M, N\right) .
$$

In Theorem 7.5 we prove that if $S$ is defined over $\mathbb{Q}$, then $R i^{b}$ and $i^{!}$are equivalent.

Corollary 4.8 Let $i: Z \rightarrow X$ be a closed embedding, and $j: U=X \backslash Z \rightarrow X$ be the corresponding open embedding. Then for any $\Omega_{U}$-module $N$ we have $R i^{b} j_{*} N=0$.

Proof By Corollary 4.4(c), for any $\Omega_{Z}$-module $M$ we have $j^{+} i_{*} M=0$. The result follows from Theorems 4.5 and 4.7 . 


\section{Differential graded commutative algebra}

5.1 The injective hull Let $M$ be an $\Omega_{X}$-module. We say that injective $\Omega_{X}^{\mathrm{gr}}$-module $E$ is an injective hull of $M^{\mathrm{gr}}$, if $M^{\mathrm{gr}} \subset E$, and every nonzero submodule of $E$ intersects $M^{\mathrm{gr}}$ non-trivially. The injective hull of a graded module exists and is unique up to an isomorphism [4]. We say that injective $\Omega_{X}$-module $E$ is an injective hull of $M$ if the graded module $E^{\mathrm{gr}}$ is the injective hull of $M^{\mathrm{gr}}$.

Proposition For any $\Omega_{X}$-module $M$ there exists an injective hull $E(M)$ of $M$.

Proof Take an injective $\Omega_{X}$-module $I$ which contains $M$ as a submodule. Let $E^{\text {gr }}$ be the injective hull of the graded module $M^{\mathrm{gr}}$ in $I^{\mathrm{gr}}$. The module $E^{\mathrm{gr}}$ generates an $\Omega_{X}$-submodule $E_{1}$ of $I$. Since the hull $E^{\mathrm{gr}}$ is injective, $E_{1}^{\mathrm{gr}} \cong E^{\mathrm{gr}} \oplus E_{2}$ for some graded $\Omega_{X}^{\mathrm{gr}}$-submodule $E_{2}$ of $E_{1}^{\mathrm{gr}}$. The $\Omega_{X}^{\mathrm{gr}}$-module $E_{2}$ can be generated by (some set of) linear combinations of $d e$ for $e \in E^{\mathrm{gr}}$, so $E_{2}$ is an $\Omega_{X}$-submodule of $E_{1}$. Put $E(M)=E_{1} / E_{2}$.

Remark By definition, the graded module $E(M)^{\text {gr }}$ is unique up to isomorphism. However, here is an example of an $\Omega_{X}$-module $M$ such that $E(M)^{\text {gr }}$ has two different differentials compatible with differential on $M$. Suppose $X$ is affine. Let $M$ be an $\Omega_{X}$-module such that $d f \cdot M=0$ for some $f \in \mathcal{O}_{X}(X)$. For example, $M=d f \wedge \Omega_{X}$. By the proposition, there exists a module $E(M)$ with some differential $d$. Then $d+d f$ is the second differential on $E(M)$, and $d m=(d+d f) m$ for any $m \in M(X)$.

5.2 The stupid filtration Let $M$ be an $\Omega_{X}$-module. Define the stupid filtration st. on $M$ by the formula st ${ }_{i} M=\Omega_{X}^{i} M$. Clearly, st $_{i} M$ is an $\Omega_{X}$-submodule of $M$. The associated graded module $\mathrm{Gr}^{\text {st }} M$ is a DG-module over $\mathrm{Gr}^{\text {st }} \Omega_{X}=\Omega_{X}^{\mathrm{gr}}$. In particular, the differential on $\mathrm{Gr}^{\mathrm{st}} M$ is $\Omega_{X}^{\mathrm{gr}}$-linear. We define the stupid filtration for $\Omega_{X}^{\mathrm{gr}}$-modules in the same way.

Proposition 5.3 Let $M$ be an $\Omega_{X}^{\mathrm{gr}}$-module. Suppose that $\mathcal{T}_{\Omega_{X}}^{1}{ }_{\Omega_{X}}^{\mathrm{gr}}\left(\mathcal{O}_{X}, M\right)=0$. Then $\mathrm{Gr}_{i}^{\mathrm{st}} M \cong \Omega_{X}^{i} \otimes_{\Omega_{X}^{\mathrm{gr}}} M$, and $\mathrm{Gr}^{\mathrm{st}} M \cong \Omega_{X}^{\mathrm{gr}} \otimes_{\mathcal{O}_{X}} \mathrm{Gr}_{0}^{\mathrm{st}} M$.

Proof Take two short exact sequences of perfect $\Omega_{X}^{\mathrm{gr}}$-modules

$$
\begin{aligned}
& 0 \rightarrow \mathrm{st}_{i} \Omega_{X} \rightarrow \Omega_{X} \rightarrow \Omega_{X} / \mathrm{st}_{i} \Omega_{X} \rightarrow 0, \\
& 0 \rightarrow \mathrm{st}_{i+1} \Omega_{X} \rightarrow \mathrm{st}_{i} \Omega_{X} \rightarrow \Omega_{X}^{i} \rightarrow 0 .
\end{aligned}
$$

Take the tensor product over $\Omega_{X}^{\mathrm{gr}}$ of these sequences with $M$. We get that $\mathrm{st}_{i} M \cong$ $\mathrm{st}_{i} \Omega_{X} \otimes_{\Omega_{X}^{\mathrm{gr}}} M$ and that

$$
\mathrm{Gr}_{i}^{\mathrm{st}} M \cong \Omega_{X}^{i} \otimes_{\Omega_{X}^{\mathrm{gr}}} M \cong \Omega_{X}^{i} \otimes_{\mathcal{O}_{X}} \mathrm{Gr}_{0}^{\mathrm{st}} M
$$

Corollary 5.4 The functor $\mathrm{Gr}^{\mathrm{st}}$ is exact on the category of thick $\Omega_{X}^{\mathrm{gr}}$-modules. 
Proof $\mathrm{Gr}_{0}^{\mathrm{st}} \cong \mathcal{O}_{X} \otimes_{\Omega_{X}^{\mathrm{gr}}}-$ and $\Omega_{X}^{i} \otimes_{\mathcal{O}_{X}}-$ are exact on $\Omega_{X}-\mathrm{qcoh}$ thick

Theorem 5.5 The following conditions on an $\Omega_{X}^{\mathrm{gr}}$-module A are equivalent:

(a) A is thick;

(b) $\mathcal{T o r}_{\Omega_{X}^{\mathrm{gr}}}^{1}\left(\mathcal{O}_{X}, A\right)=0$;

(c) $\mathrm{Gr}^{\mathrm{st}} A$ is (co)induced;

(d) $\mathrm{Gr}^{\mathrm{st}} A$ is thick.

Proof By Theorem 3.5, (a) $\Rightarrow$ (b) and (c) $\Rightarrow(d)$. By Proposition 5.3, (b) $\Rightarrow$ (c). We now prove $(\mathrm{d}) \Rightarrow$ (a). Take a finite resolution for $\mathrm{Gr}^{\text {st }} A$ by modules which are locally free over $\Omega_{X}^{\mathrm{gr}}$

$$
0 \rightarrow P_{n} \rightarrow \cdots \rightarrow P_{0} \rightarrow \mathrm{Gr}^{\mathrm{st}} A \rightarrow 0
$$

Let $P_{0}=Q_{0} \otimes \mathcal{O}_{X} \Omega_{X}^{\mathrm{gr}}$. Then there is a surjection $\varphi: Q_{0} \cong \mathrm{Gr}_{0}^{\mathrm{st}} P_{0} \rightarrow \mathrm{Gr}_{0}^{\mathrm{st}} A$. For any point of $X$ there exists a neighborhood $U$ such that the restriction $\varphi_{\mid U}$ lifts to a surjection of $\Omega_{U}^{\mathrm{gr}}$-modules $P_{0 \mid U}^{\mathrm{gr}} \rightarrow A_{\mid U}^{\mathrm{gr}}$. This gives a surjection of $\Omega_{U}$-modules $\widetilde{\varphi}: \widetilde{P}_{0}=\mathrm{DG}^{+}\left(Q_{0 \mid U}\right) \rightarrow A_{\mid U}$, and the projective dimension

$$
\operatorname{pd}_{\mathcal{O}_{U}} \mathrm{Gr}^{\mathrm{st}}(\operatorname{ker} \widetilde{\varphi})=\operatorname{pd}_{\mathcal{O}_{U}} \mathrm{Gr}^{\mathrm{st}} A-1
$$

Similarly, we can make $U$ smaller and construct a finite resolution for $A_{\mid U}$ by thick $\Omega_{U}$-modules

$$
0 \rightarrow \widetilde{P}_{n} \rightarrow \cdots \rightarrow \widetilde{P}_{0} \rightarrow A_{\mid U} \rightarrow 0
$$

It follows that $A_{\mid U}$ is thick. Thus for any $i>0$ the sheaf $\mathcal{T}_{\mathcal{O}}{ }_{\Omega_{X}^{\mathrm{gr}}}^{\mathrm{gr}}\left(\mathcal{O}_{X}, A\right)$ is trivial.

Example 5.6 Here is an example of a thick $\Omega_{X}^{\mathrm{gr}}$-module $M$ which is not induced. Let $X=\operatorname{Spec} k[t]$, and let $\delta=k[t] / t k[t]$ be a skyscraper module. Put $M=k[t] \oplus$ $k[t] d t / t \oplus \delta d t$, where $k[t] d t / t$ is the submodule of $k(t) d t$ generated over $k[t]$ by $d t / t$. The action of $t$ is natural, and to define the action of $d t$ one should write $k[t] d t / t$ as the sum $k[t] d t \oplus \delta$. We see that $\mathrm{Gr}^{\mathrm{st}} M \cong \Omega_{X}^{\mathrm{gr}} \oplus \delta \otimes_{\mathcal{O}_{X}} \Omega_{X}^{\mathrm{gr}}$. Thus $M$ is thick and is not induced.

\section{Kashivara theorem for $\Omega_{X}$-modules}

6.1 For an open subset $U \subset X$ we denote by $j_{U}: U \rightarrow X$ the corresponding open embedding. Recall that $j_{U}^{!} \cong j_{U}^{\bullet}(-) \otimes_{j_{U}^{\bullet} \mathcal{O}_{X}} \mathcal{O}_{U}$. This justifies the following notation: $M_{\mid U}=j_{U}^{!} M$, where $M$ is an $\Omega_{X}$-module.

Lemma Let $M$ be an $\Omega_{X}$-module, and let $U$ and $V$ be open subsets of $X$. Then we have the Mayer-Vietoris triangle in $\mathrm{D}^{\mathrm{co}}\left(\Omega_{X}\right.$-qcoh $)$

$$
j_{U \cup V *} j_{U \cup V}^{!} M \rightarrow j_{U *} j_{U}^{!} M \oplus j_{V *} j_{V}^{!} M \rightarrow j_{U \cap V *} j_{U \cap V}^{!} M \rightarrow
$$


Proof Let $I$ be an injective representative for $M$. Then the sequence of $\Omega_{X}$-modules

$$
0 \rightarrow j_{U \cup V *} I_{\mid U \cup V} \rightarrow j_{U *} I_{\mid U} \oplus j_{V *} I_{\mid V} \rightarrow j_{U \cap V *} I_{\mid U \cap V} \rightarrow 0
$$

is exact since $I$ is flabby.

It follows that coacyclicity is a local property.

Corollary Let $M$ be an $\Omega_{X}$-module. Suppose there is a finite covering $U$ of $X$ such that for any $U \in \mathcal{U}$ the restriction $M_{\mid U}$ is coacyclic. Then $M$ is coacyclic.

6.2 Let $i: Z \rightarrow X$ be a closed embedding, and $j: U=X \backslash Z \rightarrow X$ be the corresponding open embedding. Define the functor of "sections with support in $Z$ "

$$
\Gamma_{Z}^{X}: \Omega_{X}-\mathrm{qcoh} \rightarrow \Omega_{X}-\mathrm{qcoh}
$$

as follows. Let $J_{Z} \subset \mathcal{O}_{X}$ be the ideal sheaf of $Z$. Then the DG-ideal sheaf of $Z$ is the DG-ideal $J \subset \Omega_{X}$ generated by $J_{Z}$ and $d J_{Z}$. For an $\Omega_{X}$-module $M$ put

$$
\Gamma_{Z}^{X}(M)=\underset{m}{\lim } \mathcal{H} o \Omega_{\Omega_{X}}\left(\Omega_{X} / J^{m}, M\right)
$$

In fact, $\Gamma_{Z}^{X}(M) \cong \underline{\lim }_{m} \mathcal{H}_{\text {omm }} \mathcal{O}_{X}\left(\mathcal{O}_{X} / J_{Z}^{m}, M\right)$. Using the argument similar to [5, III.3.2] one proves that for an injective $\Omega_{X}$-module $I$ the module $\Gamma_{Z}^{X}(I)$ is also injective. Clearly, $\Gamma_{Z}^{X}$ is exact on injective $\Omega_{X}$-modules. Thus we can define the derived functor $R \Gamma_{Z}^{X}: \mathrm{D}^{\mathrm{co}}\left(\Omega_{X}\right.$-qcoh $) \rightarrow \mathrm{D}^{\mathrm{co}}\left(\Omega_{X}\right.$-qcoh $)$ using injective resolutions. When $X$ is fixed we often write $\Gamma_{Z}$ for $\Gamma_{Z}^{X}$.

Proposition For any $\Omega_{X}$-module $M$ there is a functorial distinguished triangle in $\mathrm{D}^{\mathrm{co}}\left(\Omega_{X}-\mathrm{qcoh}\right)$ :

$$
R \Gamma_{Z}(M) \rightarrow M \rightarrow j_{*} j^{!} M \rightarrow
$$

Proof We have to prove that for an injective $\Omega_{X}$-module $I$ there is an exact triple

$$
0 \rightarrow \Gamma_{Z}(I) \rightarrow I \rightarrow j_{*} j^{!} I \rightarrow 0
$$

The left morphism is the natural inclusion, and the right morphism comes from Theorem 4.5. Since $I$ is injective, the triple is left and right exact. Now we prove that the triple is exact in the middle. We may assume that $X$ is affine. Let $m \in I(X)$. If the restriction of $m$ on $U$ is zero, then for any $f \in J_{Z}$ there exist $k(f)$ such that $f^{k(f)} m=0$. Since $J_{Z}$ is finitely generated there exists $k$ such that $J^{k} m=0$, i.e. $m \in \Gamma_{Z}(I)$.

Theorem 6.3 Assume that $S$ is defined over $\mathbb{Q}$, i.e. all natural numbers are invertible in $S$. If $Z$ is smooth, then for any $\Omega_{X}$-module $M$ we have $i_{*} R i^{b}(M) \cong R \Gamma_{Z}(M)$ in $\mathrm{D}^{\mathrm{co}}\left(\Omega_{X}-\mathrm{qcoh}\right)$. 
Proof Take an injective resolution $I$ of $M$. Then $i_{*} i{ }^{b}(I) \cong \mathcal{H} m_{\Omega_{X}}\left(i_{*} \Omega_{Z}, I\right)$ is a submodule of $\Gamma_{Z}(I)$. This gives a morphism of functors. It is enough to prove that this morphism is equivalence when $X$ is affine, and $J_{Z}$ is generated by a regular sequence $f_{1}, \ldots, f_{r}$.

Lemma 6.3.1 Let $a: Z_{1} \rightarrow X$ and $Z_{2} \subset Z_{1}$ be closed embeddings. Then

$$
R \Gamma_{Z_{2}}^{Z_{1}} \circ R a^{b} \cong R a^{b} \circ R \Gamma_{Z_{2}}^{X}
$$

Proof Apply both sides to $I$. The module $E=\Gamma_{Z_{2}}^{X}(I)$ is injective, thus $a^{b}(E)$ computes the right hand side. By Proposition 6.2, we have the short exact sequence of $\Omega_{X}$-modules

$$
0 \rightarrow E \rightarrow I \rightarrow j_{*} j^{!} I \rightarrow 0
$$

where $j: X \backslash Z_{2} \rightarrow X$ is the natural open embedding. Apply the functor $\Gamma_{Z_{2}}^{Z_{1}} \circ a^{b}$ to this exact sequence. By Corollary $4.8, a^{b} j_{*} j^{!} I \cong 0$, and it is easy to check that $\Gamma_{Z_{2}}^{Z_{1}} \circ a^{b}(E) \cong a^{b}(E)$. The lemma follows.

By the lemma, we may assume that the ideal of $Z$ in $\mathcal{O}_{X}$ is generated by a single regular function $f$.

Let $N=\Gamma_{Z}(I)$, and let $N_{0} \cong i_{*} i^{b}(I)$ be the submodule of $N$ annihilated by $f$ and $d f$. Put

$$
N_{k}=\left\{m \in N: f^{k} m \in N_{0}\right\}
$$

By Lemma 6.3.2 below, $N_{k}$ is an $\Omega_{X}$-module. Clearly, $N \cong \lim _{k} N_{k}$. We claim that for $k>0$ the module $N_{k} / N_{k-1}$ is contractible. Then by Lemma 3.2(c), $N$ is coisomorphic to $N_{0}$. We prove the claim.

Lemma 6.3.2 If $m \in N_{k}$, then $d m \in N_{k}$.

Proof We prove that $f^{k} d m \in N_{0}$, i.e. $f\left(f^{k} d m\right)=0$, and $d f\left(f^{k} d m\right)=0$. This is clear from the relations

$$
\begin{aligned}
& 0=d\left(f^{k+1} m\right)=(k+1) f^{k} d f \cdot m+f^{k+1} d m, \\
& 0=d\left(f^{k} d f \cdot m\right)=-f^{k} d f \cdot d m
\end{aligned}
$$

Lemma 6.3.3 If $d f \cdot m=0$ for some $m \in I$, then there exists $\beta \in I$ such that $m=d f \cdot \beta$.

Proof Recall that $f$ is regular, and $I$ is injective. Define the morphism $\varphi: d f \wedge \Omega_{X} \rightarrow$ $I$ by $\varphi(d f \wedge \omega)=\omega \cdot m$. This definition is correct since $f$ is regular. We can lift $\varphi$ to a morphism $\tilde{\varphi}: \Omega_{X} \rightarrow I$. Put $\beta=\widetilde{\varphi}(1)$.

Lemma 6.3.4 Suppose that $m \in N_{k}$. Then there exists $\alpha \in N_{k}$ such that $f^{k} m=$ $f^{k-1} d f \cdot \alpha$, and $f^{k} \alpha=0$. 
Proof Put $m^{\prime}=f^{k} m \in N_{0}$. Then $d f \cdot m^{\prime}=0$, and, by Lemma 6.3.3, there exists $\beta \in I$ such that $m^{\prime}=d f \cdot \beta$. By assumption,

$$
f d f \cdot \beta=f m^{\prime}=0 .
$$

As before, there exists $\gamma \in I$ such that $f \beta=f d f \cdot \gamma$. Put $\alpha^{\prime}=\beta-d f \cdot \gamma$. Then $f \alpha^{\prime}=0$, and $m^{\prime}=d f \cdot \alpha^{\prime}$. There exists $\alpha \in I$ such that $f^{k-1} \alpha=\alpha^{\prime}$. Thus $f^{k} \alpha=0$, and $\alpha \in N_{k}$.

We define an endomorphism $s$ on $N_{k} / N_{k-1}$ as follows. Take $m \in N_{k}$. Then, by Lemma 6.3.4, there exists $\alpha \in N_{k}$ such that $f^{k} m=f^{k-1} d f \cdot \alpha$. Let $s(m)$ be the image of $\alpha$ in $N_{k} / N_{k-1}$. Clearly, $s(m)$ is independent on the choice of $\alpha$, and $s$ is a morphism of $\Omega_{X}^{\mathrm{gr}}$-modules of degree -1 . Let us prove that $d s+s d=-k$. From the relation $f^{k} m=f^{k-1} d f \cdot \alpha$ we get

$$
f^{k} d m=f^{k-1} d f(-d \alpha-k m)
$$

It follows that $s(d m)=-d s(m)-k m$, and $-s / k$ is a contracting homotopy for $N_{k} / N_{k-1}$.

Corollary 6.4 Assume that $S$ is defined over $\mathbb{Q}$. Let I be an injective $\Omega_{Z}$-module. Then an injective hull $E$ of $i_{*} I$ is co-isomorphic to $i_{*} I$.

Proof Note that $E=\Gamma_{Z}(E)$, because it is true for the graded module $E^{\mathrm{gr}}$. By Theorem $6.3, E \cong i_{*} i^{b}(E)$ in $\mathrm{D}^{\mathrm{co}}\left(\Omega_{X}\right.$-qcoh). We prove that $i^{b}(E)$ is isomorphic to $I$ over $\Omega_{Z}$. Since $I$ is injective, it is a direct summand of $i^{b}(E)$ as a graded module, i.e. $i^{b}(E)^{\mathrm{gr}} \cong I^{\mathrm{gr}} \oplus I^{\prime}$ for some $\Omega_{Z}^{\mathrm{gr}}$-module $I^{\prime}$. Thus $i_{*} I^{\prime}$ is a submodule of $E^{\mathrm{gr}}$ such that $i_{\bullet} I^{\prime} \cap i_{\bullet} I=0$. Since $E^{\mathrm{gr}}$ is an injective hull of $i_{\bullet} I^{\mathrm{gr}}$, we conclude that $i_{\bullet} I^{\prime}=0$. The functor $i_{\bullet}$ is full and faithful, thus $I^{\prime}=0$.

Theorem 6.5 (Kashivara theorem) Assume that $S$ is defined over $\mathbb{Q}$. Let $\mathrm{D}_{Z}^{\mathrm{co}}\left(\Omega_{X}\right.$-qcoh) be the full subcategory of $\mathrm{D}^{\mathrm{co}}\left(\Omega_{X}\right.$-qcoh) of $\Omega_{X}$-modules with support on $Z$, and $\mathrm{D}_{Z}^{\mathrm{abs}}\left(\Omega_{X}\right.$-coh) be the corresponding category of coherent $\Omega_{X}$-modules. Then the functor $i_{*}$ gives an equivalence of $\mathrm{D}^{\mathrm{co}}\left(\Omega_{Z}\right.$-qcoh $)$ with $\mathrm{D}_{Z}^{\mathrm{co}}\left(\Omega_{X}\right.$-qcoh $)$, and of $\mathrm{D}^{\mathrm{abs}}\left(\Omega_{Z}\right.$-coh $)$ with $\mathrm{D}_{Z}^{\mathrm{abs}}\left(\Omega_{X}\right.$-coh). In particular, $i_{*}$ is full and faithful.

Let $M$ be an object of $\mathrm{D}_{Z}^{\mathrm{co}}\left(\Omega_{X}\right.$-qcoh). By Theorem 6.3 and Proposition 6.2, we have the distinguished triangle in $\mathrm{D}^{\mathrm{co}}\left(\Omega_{X}\right.$-qcoh $)$

$$
i_{*} R i^{b}(M) \rightarrow M \rightarrow j_{*} j^{+} M \rightarrow
$$

By assumption, $j^{+} M=0$, thus $i_{*} R i^{b} \cong \operatorname{Id}_{D_{Z}^{\text {co }}\left(\Omega_{X} \text {-qcoh }\right)}$, and by Corollary 6.4 , $R i^{b} i_{*} \cong \operatorname{Id}_{\mathrm{D}^{\mathrm{co}}\left(\Omega_{\mathrm{Z}} \text {-qcoh }\right)}$. For a coherent $\Omega_{Z}$-module $M$ the module $i_{*} M$ is also coherent. Conversely, any coherent $\Omega_{X}$-module $M$ with support in $Z$ has a finite filtration such that the associated graded module is the direct image $i_{*} M^{\prime}$ of a coherent $\Omega_{Z}$-module $M^{\prime}$. This is enough, since $\mathrm{D}^{\mathrm{abs}}\left(\Omega_{X}-\mathrm{coh}\right)$ is a full subcategory of $\mathrm{D}^{\mathrm{co}}\left(\Omega_{X^{-}} \mathrm{qcoh}\right)[11$, 3.11]. 
Theorem 6.6 (base change) Assume that $S$ is defined over $\mathbb{Q}$. Let $f: X \rightarrow Y$ and $g: Z \rightarrow Y$ be two morphisms of smooth schemes over $S$. Consider the Cartesian square

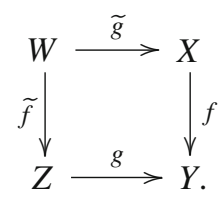

Assume that $W=X \times{ }_{Y} Z$ is smooth over $S$. Then there exists an isomorphism

$$
g^{!} \circ f_{*} \cong \widetilde{f}_{*} \circ \widetilde{g}^{!}: \mathrm{D}^{\mathrm{co}}\left(\Omega_{X}-\mathrm{qcoh}\right) \rightarrow \mathrm{D}^{\mathrm{co}}\left(\Omega_{Z}-\mathrm{qcoh}\right)
$$

The proof is the same as in $[6,1.7 .3]$.

\section{Trace map for a closed embedding}

7.1 Čech resolution Let $\mathcal{U}=\left\{U_{i}\right\}_{i \in I}$ be a finite open covering of $X$, where $I=$ $\{0, \ldots, N\}$ is a finite ordered set. Let $M$ be an $\Omega_{X}$-module. We define the Čech resolution $C^{\bullet}(\mathcal{U}, M)$ of $M$ as follows:

$$
C^{n}(\mathcal{U}, M)=\bigoplus_{J \subset I} j_{J *} j_{J}^{+} M
$$

where $J$ is a subset of $I$ of order $|J|=n+1$, and $j_{J}$ is the inclusion $\bigcap_{i \in J} U_{i} \rightarrow X$. The closed morphism $\partial_{n}: C^{n}(\mathcal{U}, M) \rightarrow C^{n+1}(\mathcal{U}, M)$ is defined in the usual way. Put $C(\mathcal{U}, M)=\operatorname{Tot}^{\oplus} C^{\bullet}(\mathcal{U}, M)$. The natural closed morphisms $M \rightarrow j_{i *} M_{\mid U_{i}}$ induce the natural inclusion morphism $M \rightarrow C^{0}(\mathcal{U}, M) \rightarrow C(\mathcal{U}, M)$.

Lemma The closed morphism $M \rightarrow C(\mathcal{U}, M)$ is a co-isomorphism. Moreover, its cone is absolutely acyclic.

Proof The sequence of $\Omega_{X}^{\mathrm{gr}}$-modules

$$
0 \rightarrow M^{\mathrm{gr}} \rightarrow C^{0}(\mathcal{U}, M)^{\mathrm{gr}} \rightarrow \cdots \rightarrow C^{|I|}(\mathcal{U}, M)^{\mathrm{gr}} \rightarrow 0
$$

is exact, because it is the Čech resolution of the graded quasi-coherent $\mathcal{O}_{X}$-module $M^{\mathrm{gr}}$. The $\Omega_{X}$-module cone $(M \rightarrow C(\mathcal{U}, M))$ is the total object of the sequence

$$
0 \rightarrow M \rightarrow C^{0}(\mathcal{U}, M) \rightarrow \cdots \rightarrow C^{|I|}(\mathcal{U}, M) \rightarrow 0 .
$$

It is absolutely acyclic, since we can cut this sequence into short exact sequences. The lemma follows.

Theorem Suppose $\mathcal{U}$ is a finite affine covering of $X$. Then $f_{*}(M)$ is co-isomorphic to $f_{\bullet}(C(\mathcal{U}, M))$. 
Proof Let $N$ be an injective resolution of $M$. Then $j_{J}^{+}(N)$ is flabby for any $J \subset I$, thus $C(\mathcal{U}, N)$ is flabby too, and $f_{*}(M)$ is co-isomorphic to $f_{\bullet}(N)$. By the lemma, $f_{\bullet}(N)$ is co-isomorphic to $f_{\bullet} C(\mathcal{U}, N)$. There is the natural morphism $\varphi: C(\mathcal{U}, M) \rightarrow C(\mathcal{U}, N)$ with coacyclic cone. Since the morphism $g=f \circ j_{J}$ is affine, the functor $g_{\bullet}$ is exact, and $g_{\bullet} j_{J}^{+}(N)$ is co-isomorphic to $g_{\bullet} j_{J}^{+}(M)$. It follows that $\varphi$ induces a morphism $f_{\bullet} C(\mathcal{U}, M) \rightarrow f_{\bullet} C(\mathcal{U}, N)$ with coacyclic cone.

7.2 Gluing morphisms in $\mathrm{D}^{\mathrm{co}}\left(\Omega_{X}\right.$-qcoh $)$ In this section we prove a counterpart of [1, 3.2.2] for $\Omega_{X}$-modules.

Theorem Let $M$ and $N$ be $\Omega_{X}$-modules. Suppose that $M$ is coherent. Let $\mathcal{U}$ be a finite affine covering of $X$. Assume that $\operatorname{Hom}_{D^{\mathrm{co}}\left(\Omega_{V} \text {-qcoh) }\right.}\left(M_{\mid V}, N_{\mid V}[j]\right)=0$ for all affine $V \subset U \in \mathcal{U}$ and $j<0$. Then

$$
\mathcal{H o m}_{\mathrm{D}^{\mathrm{co}}\left(\Omega_{X}-\mathrm{qcoh}\right)}(M, N): V \mapsto \operatorname{Hom}_{\mathrm{D}^{\mathrm{co}}\left(\Omega_{V}-\mathrm{qcoh}\right)}\left(M_{\mid V}, N_{\mid V}\right)
$$

is a sheaf.

Proof It is enough to prove that sections over $X$ can be glued of sections over the covering $\mathcal{U}$. We may assume that $N$ is injective. Define the presheaf

$$
\mathcal{H}^{q}: V \mapsto \operatorname{Hom}_{D^{\mathrm{co}}\left(\Omega_{V}-\mathrm{qcoh}\right)}\left(M_{\mid V}, N_{\mid V}[q]\right) .
$$

We have to prove that $\mathcal{H}^{0}$ is a sheaf. By Proposition 4.3,

$$
\mathcal{H}^{q}(V) \cong H^{q}\left(S, f_{\mid V *} \mathcal{H o m}_{\Omega_{V}}\left(M_{\mid V}, N_{\mid V}\right)\right),
$$

where $f: X \rightarrow S$ is the morphism to the base scheme, and $f_{\mid V}$ is the restriction to $V$. By Theorem 7.1, we have the Čech spectral sequence

$\left.H^{p}\left(\mathcal{U}, \mathcal{H}^{q}\right)\right) \Longrightarrow H^{p+q}\left(S, f_{*} \mathcal{H o m}_{\Omega_{X}}(M, N)\right) \cong \operatorname{Hom}_{D^{\mathrm{co}}\left(\Omega_{X} \text {-qcoh }\right)}(M, N[p+q])$.

By assumption, $\left.H^{p}\left(\mathcal{U}, \mathcal{H}^{q}\right)\right)=0$ for $q<0$. It follows that $H^{0}\left(\mathcal{U}, \mathcal{H}^{0}\right) \cong$ $\operatorname{Hom}_{D^{\mathrm{co}}\left(\Omega_{X} \text {-qcoh }\right)}(M, N)$.

7.3 Koszul resolution Let $i: Z \rightarrow X$ be a closed embedding of affine smooth schemes over $S$. Suppose that $Z$ is a complete intersection in $X$. In this section we construct an explicit perfect resolution for $\Omega_{Z}$ over $\Omega_{X}$.

Theorem There exists a perfect $\Omega_{X}$-module $K$ and a non-canonical co-isomorphism $K[n] \rightarrow i_{*} \Omega_{Z}$

Lemma 7.3.1 Suppose that the ideal of $Z$ in $\mathcal{O}_{X}$ is generated by $f_{1}, \ldots, f_{n} \in$ $\mathcal{O}_{X}$, where $n=\operatorname{dim} X-\operatorname{dim} Z$. Let $P_{Z}$ be the submodule of $\Omega_{X}$ generated by $d f_{1} \wedge \cdots \wedge d f_{n}$, and let $I_{Z}$ be the submodule $f_{1} P_{Z}+\cdots+f_{n} P_{Z}$. Then $P_{Z}$ is perfect, and $i_{*} \Omega_{Z} \cong P_{Z} / I_{Z}[n]$. 
Proof Locally there exist functions $z_{1}, \ldots, z_{s} \in \mathcal{O}_{X}$ such that $d z_{1}, \ldots, d z_{s}$, $d f_{1}, \ldots, d f_{n}$ generate $\Omega_{X}^{1}$ over $\mathcal{O}_{X}$. Thus $P_{Z}$ is a direct summand in $\Omega_{X}$ and perfect. We have the natural surjective morphism $P_{Z}[n] \rightarrow i_{*} \Omega_{Z}$ given by $d f_{1} \wedge \cdots \wedge d f_{n} \mapsto$ 1. Clearly, its kernel is $I_{Z}$.

Lemma 7.3.2 Let $L_{\bullet}$ be the Koszul resolution of $\mathcal{O}_{Z}$ over $\mathcal{O}_{X}$. Take the complex of $\Omega_{X}^{\mathrm{gr}}$-modules $K_{\bullet}=L_{\bullet} \otimes_{\mathcal{O}_{X}} P_{Z}$. Then the induced morphism $\delta_{m}: K_{m} \rightarrow K_{m-1}$ is a closed morphism of $\Omega_{X}$-modules.

Proof By definition of the Koszul complex, $L_{\bullet} \cong \bigwedge^{\bullet} L_{1}$ is a DG-algebra over $\mathcal{O}_{X}$ generated by $L_{1}$, where $L_{1} \cong \bigoplus_{i=1}^{n} v_{i} \mathcal{O}_{X}$ for some $v_{i} \in L_{1}$ and the differential is given by $v_{i} \mapsto f_{i}$. Thus $K_{0} \cong P_{Z}$, and $K_{1} \cong \bigoplus_{i=1}^{n} v_{i} P_{Z}$. The differential $\delta_{m}$ on $K_{\bullet}$ comes from the formula

$$
\delta_{1}\left(v_{i} \otimes d f_{1} \wedge \cdots \wedge d f_{n}\right)=f_{i} d f_{1} \wedge \cdots \wedge d f_{n} .
$$

Now it is clear that $\delta_{m}$ is a closed morphism of $\Omega_{X}$-modules.

Proof of Theorem 7.3 Clearly, the sequence of $\Omega_{X}$-modules

$$
0 \rightarrow K_{n} \rightarrow \cdots \rightarrow K_{1} \rightarrow K_{0} \cong P_{Z} \rightarrow i_{*} \Omega_{Z}[-n] \rightarrow 0
$$

is exact. Put $K=\operatorname{Tot}^{\oplus}\left(K_{\bullet}\right)$.

Theorem 7.4 Suppose that $S$ is a $\mathbb{Q}$-scheme. Then there is a natural co-isomorphism tr: $R i^{b}\left(\Omega_{X}\right) \cong \Omega_{Z}[2 \operatorname{dim} X-2 \operatorname{dim} Z]$.

Proof Apply $i_{*}$ to both sides. By Theorem 6.5, it is enough to prove that

$$
R \mathcal{H o m}_{\Omega_{X}}\left(i_{*} \Omega_{Z}, \Omega_{X}\right) \cong i_{*} \Omega_{Z}[2 \operatorname{dim} X-2 \operatorname{dim} Z]
$$

We construct the isomorphism locally and then use Theorem 7.2.

Assume that $X$ is affine and the ideal of $Z$ in $\mathcal{O}_{X}$ is generated by $f_{1}, \ldots, f_{n} \in$ $\mathcal{O}_{X}$, where $n=\operatorname{dim} X-\operatorname{dim} Z$. Let $K[n]$ be the Koszul resolution for $i_{*} \Omega_{Z}$. By Theorem 4.2,

$$
R \mathcal{H o m}_{\Omega_{X}}\left(K[n], \Omega_{X}\right) \cong \mathcal{H o m}_{\Omega_{X}}\left(K[n], \Omega_{X}\right)
$$

We use the notation from the proof of Lemma 7.3.2. By definition, $K_{1} \cong \bigoplus_{i=1}^{n} v_{i} P_{Z}$. Hence $K_{n} \cong P_{Z}$ is generated by $v_{1} \wedge \cdots \wedge v_{n}$. Moreover, $\mathcal{H}_{0} \boldsymbol{m}_{X}\left(P_{Z}, \Omega_{X}\right)$ is isomorphic to $P_{Z}[n]$ non-canonically. Thus the complex of $\Omega_{X}$-modules $\check{K}^{\bullet}=$ $\mathcal{H} m_{\Omega_{X}}\left(K_{\bullet}[n], \Omega_{X}\right)$ is isomorphic to $K_{\bullet}$. The module $\check{K}^{n}[n]=\mathcal{H}_{Z} m_{\Omega_{X}}\left(K_{n}, \Omega_{X}\right) \cong$ $P_{Z}$ has the generator $u$ given by

$$
v_{1} \wedge \cdots \wedge v_{n} \mapsto d f_{1} \wedge \cdots \wedge d f_{n}
$$


As in Lemma 7.3.1, we define the morphism $\check{K}^{n}[n] \rightarrow \Omega_{Z}$ such that $u$ goes to $1 \in \Omega_{Z}$. This gives a co-isomorphism

$$
\psi_{X}: \check{K}=R \mathcal{H o m}_{\Omega_{X}}\left(i_{*} \Omega_{Z}, \Omega_{X}\right) \rightarrow i_{*} \Omega_{Z}[-2 n] .
$$

It is clear that $\psi_{X}$ does not depend on the choice of coordinates $f_{1}, \ldots, f_{n}$.

We proved the theorem locally and now turn to the global situation. We have to find a co-isomorphism of $\check{K}=R \mathcal{H o m}_{\Omega_{X}}\left(i_{*} \Omega_{Z}, \Omega_{X}\right)$ and $i_{*} \Omega_{Z}[-2 n]$. By the local result,

$$
\begin{aligned}
\operatorname{Hom}_{\mathrm{D}^{\mathrm{co}}\left(\Omega_{V} \text {-qcoh }\right)} & \left(\check{K}_{\mid V}, i_{*} \Omega_{Z \mid V}[-2 n+j]\right) \\
& \cong \operatorname{Hom}_{\mathrm{D}^{\mathrm{co}}\left(\Omega_{V} \text {-qcoh }\right)}\left(i_{*} \Omega_{Z \mid V}, i_{*} \Omega_{Z \mid V}[j]\right) \\
& \cong \operatorname{Hom}_{\mathrm{D}^{\mathrm{co}}\left(\Omega_{Z \cap V}-\mathrm{qcoh}\right)}\left(\Omega_{Z \cap V}, \Omega_{Z \cap V}[j]\right)=0
\end{aligned}
$$

for small affine open $V \subset X$ and any $j<0$. By Theorem 7.2, the presheaf $\mathcal{H} m_{\mathrm{D}^{\mathrm{co}}\left(\Omega_{X} \text {-qcoh }\right)}\left(\check{K}, i_{*} \Omega_{Z}[-2 n]\right)$ is a sheaf. Thus we can glue local morphisms $\psi_{U}$ to a global morphism $\psi \in \operatorname{Hom}_{D^{\mathrm{co}}\left(\Omega_{X} \text {-qcoh }\right)}\left(\check{K}, i_{*} \Omega_{Z}[-2 n]\right)$. This morphism is a co-isomorphism by Corollary 6.1.

Theorem 7.5 Suppose that $S$ is a $\mathbb{Q}$-scheme. Let $i: Z \rightarrow X$ be a closed embedding of smooth schemes over $S$. Then the functors $R i^{b}$ and $i$ are naturally equivalent.

Proof In Theorem 7.4 we construct the trace isomorphism for closed embedding

$$
\operatorname{tr}: R \mathcal{H o m}_{f} \bullet \Omega_{X}\left(\Omega_{Z}, f^{\bullet} \Omega_{X}\right) \cong \Omega_{Z}[2 \operatorname{dim} Z-2 \operatorname{dim} X]
$$

By Remark 3.8, any $\Omega_{X}$-module has a resolution $F$ which is locally free over $\Omega_{X}^{\mathrm{gr}}$. We have

$$
\begin{aligned}
R i^{b}(F) & =R \mathcal{H o m}_{f} \bullet \Omega_{X}\left(\Omega_{Z}, f^{\bullet}(F)\right) \cong R \mathcal{H o m}_{f} \bullet \Omega_{X}\left(\Omega_{Z}, f^{\bullet} \Omega_{X}\right) \otimes_{f} \bullet \Omega_{X} f^{\bullet}(F) \\
& \cong \Omega_{Z} \otimes_{f} \bullet \Omega_{X} f^{\bullet}(F)[2 \operatorname{dim} Z-2 \operatorname{dim} X]=i^{!}(F)
\end{aligned}
$$

\section{Coherent $\Omega$-modules}

8.1 Recall that $\mathrm{D}^{\mathrm{abs}}\left(\Omega_{X}-\mathrm{coh}\right)$ is a full subcategory of $\mathrm{D}^{\mathrm{co}}\left(\Omega_{X}\right.$-qcoh). Moreover, coherent $\Omega_{X}$-modules are compact objects of $\mathrm{D}^{\mathrm{co}}\left(\Omega_{X}\right.$-qcoh $)[11,3.11]$. Let $\mathrm{D}^{\mathrm{coh}}(X)$ be the thick envelope of $\mathrm{D}^{\text {abs }}\left(\Omega_{X}\right.$-coh $)$ in $\mathrm{D}^{\mathrm{co}}\left(\Omega_{X}\right.$-qcoh). We prove that an object $M$ of $\mathrm{D}^{\mathrm{co}}\left(\Omega_{X}\right.$-qcoh) belongs to $\mathrm{D}^{\text {coh }}(X)$ if and only if $M$ is locally coherent.

Proposition [13, Remark 1.10] Let $\mathcal{U}$ be a finite open covering of $X$ and let $M$ be an object of $\mathrm{D}^{\mathrm{abs}}\left(\Omega_{X}\right.$-coh). Then $M$ belongs to $\mathrm{D}^{\text {coh }}(X)$ if and only if for any $U \in \mathcal{U}$ the module $M_{\mid U}$ belongs to $\mathrm{D}^{\text {coh }}(U)$.

Proof We give a proof under assumption that $S$ is defined over $\mathbb{Q}$. Take $U \in \mathcal{U}$. By the Kashivara theorem, $\mathrm{D}^{\mathrm{co}}\left(\Omega_{U^{-}}\right.$qcoh $)$is equivalent to the Verdier quotient of $\mathrm{D}^{\mathrm{co}}\left(\Omega_{X}\right.$-qcoh) by $\mathrm{D}^{\mathrm{co}}\left(\Omega_{Z}\right.$-qcoh $)$. Now, by Theorem $[8,2.1 .5]$, there is an object $N$ of 
$\mathrm{D}^{\text {coh }}(X)$ and a morphism $N \rightarrow M$ such that $N_{\mid U} \rightarrow M_{\mid U}$ is a co-isomorphism. The cone $M_{1}=\operatorname{cone}(N \rightarrow M)$ is coacyclic on $U$. Take $V \in \mathcal{U}$, then $M_{1 \mid V}$ belongs to $\mathrm{D}^{\text {coh }}(V)$. We can make $V$ smaller in such a way that $Z=V \backslash(U \cap V)$ is smooth. By the Kashivara theorem, $M_{1 \mid V \cup U}$ is co-isomorphic to $i_{*} M_{Z}$, where $i: Z \rightarrow V \cup U$ is the natural inclusion, and $M_{Z}$ is coherent. We proved that $M_{\mid U \cup V}$ belongs to $\mathrm{D}^{\text {coh }}(U \cup V)$. The proposition follows by induction.

Theorem 8.2 Let $f: X \rightarrow Y$ be a morphism of smooth schemes over $S$. If $f$ is proper, and $M$ is a coherent $\Omega_{X}$-module, then $f_{*}(M)$ is isomorphic to a coherent $\Omega_{Y}$-module in $\mathrm{D}^{\mathrm{co}}\left(\Omega_{Y}\right.$-qcoh). If $f$ is smooth, and $N$ is a coherent $\Omega_{Y}$-module, then $f^{!}(N)$ is isomorphic to a coherent $\Omega_{X}$-module in $\mathrm{D}^{\mathrm{co}}\left(\Omega_{X}\right.$-qcoh).

Proof The category of coherent $\Omega_{X}$-modules is generated by coherent modules annihilated by $\Omega_{X}^{1}$. Suppose $M$ is such a module. We have to prove that $f_{*}(M)$ is coherent. The direct image can be computed using Čech resolution $C^{\bullet}(M)$. Clearly, $f_{\bullet} C^{\bullet}(M)$ is annihilated by $\Omega_{Y}^{1}$, and by Serre's theorem is quasi-isomorphic to a complex of coherent $\mathcal{O}_{Y}$-modules. This quasi-isomorphism is naturally a co-isomorphism of $\Omega_{Y^{-}}$ modules. The second part of the theorem follows from Lemma 4.4.

8.3 Duality Let $n=\operatorname{dim}_{S} X=\operatorname{dim} X-\operatorname{dim} S$ be a relative dimension of $X$ over $S$. We define the duality functor

$$
\mathrm{D}_{X}: \mathrm{D}^{\mathrm{abs}}\left(\Omega_{X}-\mathrm{coh}\right) \rightarrow \mathrm{D}^{\mathrm{abs}}\left(\Omega_{X}-\mathrm{coh}\right)
$$

by $\mathrm{D}_{X}=R \mathcal{H} \operatorname{Hom}_{\Omega_{X}}\left(-, \Omega_{X}[2 n]\right)$. Take a perfect $\Omega_{X}$-module $P$. Since $\Omega_{X}$ is coinduced the coherent module $\mathcal{H o m}_{\Omega_{X}}\left(P, \Omega_{X}[2 n]\right)$ represents $R \mathcal{H o m}_{\Omega_{X}}\left(P, \Omega_{X}[2 n]\right)$. It follows that $\mathrm{D}_{X}$ is well-defined.

Proposition $\mathrm{D}_{X}^{2} \cong \mathrm{Id}_{\mathrm{D}^{\text {abs }}\left(\Omega_{X}-\mathrm{coh}\right)}$.

Proof It is enough to prove that $\mathrm{D}_{X}^{2}$ is equivalent to the identity functor on the category of prefect $\Omega_{X}$-modules. For a perfect $\Omega_{X}$-module $P$ we have a natural transformation

$$
\begin{aligned}
\Phi: P \rightarrow \mathcal{H o m}_{\Omega_{X}} & \left(\mathcal{H o m}_{\Omega_{X}}\left(P, \Omega_{X}\right), \Omega_{X}\right) \\
& \cong \mathcal{H o m}_{\Omega_{X}}\left(\mathcal{H o m}_{\Omega_{X}}\left(P, \Omega_{X}[2 n]\right), \Omega_{X}[2 n]\right) .
\end{aligned}
$$

In fact, $\Phi$ is an isomorphism of $\Omega_{X}$-modules. We prove it locally for $P=\mathcal{O}_{X}$. In this case $\mathcal{H}_{0} \Omega_{\Omega_{X}}\left(\mathcal{O}_{X}, \Omega_{X}\right) \cong \Omega_{X}^{n}[-n]$, and $\mathcal{H o m}_{\Omega_{X}}\left(\Omega_{X}^{n}[-n], \Omega_{X}\right) \cong \mathcal{O}_{X}$.

Proposition 8.4 Let $M$ and $N$ be coherent $\Omega_{X}$-modules. Then

$$
D(M) \otimes_{\Omega_{X}}^{D} N \cong R \mathcal{H o m}_{\Omega_{X}}(M, N) .
$$

Proof Choose a perfect resolution $P$ for $M$ and a coinduced resolution $A$ for $N$, i.e. $A^{\text {gr }} \cong \mathcal{H o m}_{\mathcal{O}_{X}}\left(\Omega_{X}^{\mathrm{gr}}, L\right)$ for some graded $\mathcal{O}_{X}$-module $L$. Let $\Phi$ be the natural transformation

$$
\mathcal{H o m}_{\Omega_{X}}\left(P, \Omega_{X}\right) \otimes_{\Omega_{X}} A \rightarrow \mathcal{H o m}_{\Omega_{X}}(P, A)
$$


given by the formula $\varphi \otimes a \mapsto(p \mapsto \varphi(p) a)$, where $\varphi \in \mathcal{H}_{\text {om }} \Omega_{X}\left(P, \Omega_{X}\right), p \in P$, and $a \in A$. We claim that $\Phi$ is an isomorphism of $\Omega_{X}$-modules. Indeed, the question is local and we may assume that $P=\mathcal{O}_{X}$. In this case, $\Phi$ is an isomorphism since $A$ is coinduced. The proposition follows.

Proposition 8.5 Suppose $S$ is a $\mathbb{Q}$-scheme. Let $i: Z \rightarrow X$ be a closed embedding of smooth schemes over $S$. Then $\mathrm{D}_{X} \circ i_{*} \cong i_{*} \circ \mathrm{D}_{Z}$.

Proof By Theorems 4.7 and 7.5, for any coherent $\Omega_{Z}$-module $M$ we have

$$
\begin{aligned}
\mathrm{D}_{X}\left(i_{*} M\right) & =R \mathcal{H o m}_{\Omega_{X}}\left(i_{*} M, \Omega_{X}\left[2 \operatorname{dim}_{S} X\right]\right) \\
& \cong i_{*} R \mathcal{H} \operatorname{Hom}_{\Omega_{Z}}\left(M, i^{!} \Omega_{X}\left[2 \operatorname{dim}_{S} X\right]\right) \\
& \cong i_{*} R \mathcal{H o m}_{\Omega_{Z}}\left(M, \Omega_{Z}\left[2 \operatorname{dim}_{S} Z\right]\right)=i_{*} D_{Z}(M)
\end{aligned}
$$

8.6 Trace for a proper projection Suppose that the base scheme $S$ is defined over $\mathbb{Q}$. Let $p: \mathbb{P}^{n}=\mathbb{P}_{S}^{n} \rightarrow S$ be the natural projection from the projective space to $S$. We define the trace morphism $\operatorname{Tr}_{p}$ as the composition

$$
f_{*} \Omega_{\mathbb{P}^{n}}[2 n] \rightarrow H^{n}\left(\mathbb{P}^{n}, \Omega_{\mathbb{P}^{n}}^{n}\right) \rightarrow \mathcal{O}_{S}
$$

of the natural projection to $H^{n}\left(\mathbb{P}^{n}, \Omega_{\mathbb{P} n}^{n}\right)$ with the trace morphism defined in $[5$, Remark 10.2].

Let $g: Y \rightarrow S$ be a smooth $S$-scheme. Denote by $f: X=\mathbb{P}^{n} \times{ }_{S} Y \rightarrow Y$ and $q: X \rightarrow \mathbb{P}^{n}$ natural projections to $Y$ and $\mathbb{P}^{n}$. By the base change formula we have the trace morphism

$$
\operatorname{Tr}_{f}: f_{*} \Omega_{X}[2 \operatorname{dim} Y+2 n] \cong f_{*}\left(q^{!} \Omega_{\mathbb{P}^{n}}[2 n]\right) \cong g^{!} p_{*} \Omega_{\mathbb{P}^{n}}[2 n] \stackrel{\operatorname{Tr}_{p}}{\longrightarrow} g^{!} \mathcal{O}_{S} \cong \Omega_{Y}
$$

Lemma 8.7 Suppose $f: X \rightarrow Y$ is smooth and proper. For any $M, N \in$ $\mathrm{D}^{\mathrm{abs}}\left(\Omega_{X}\right.$-coh) there is a natural morphism

$$
f_{*} R \mathcal{H o m}_{\Omega_{X}}(M, N) \rightarrow R \mathcal{H o m}_{\Omega_{X}}\left(f_{*} M, f_{*} N\right) .
$$

Proof By Theorem 8.2, $f_{*} M$ is coherent, and the right hand side is well-defined. By Theorem 4.5, we have an adjunction morphism $f^{+} f_{*} M \rightarrow M$, and the desired morphism is the composition 


$$
f_{*} R \mathcal{H o m}_{\Omega_{X}}(M, N) \rightarrow f_{*} R \mathcal{H o m}_{\Omega_{X}}\left(f^{+} f_{*} M, N\right) \rightarrow R \mathcal{H} \operatorname{Hom}_{\Omega_{X}}\left(f_{*} M, f_{*} N\right)
$$

Theorem 8.8 Let $f: X \rightarrow Y$ be a proper morphism of smooth schemes over $S$. Then $\mathrm{D}_{Y} \circ f_{*} \cong f_{*} \circ \mathrm{D}_{X}$.

Proof The morphism $f$ is a composition of a closed embedding and a proper projection. By Proposition 8.5, we have to prove the theorem for the projection $f: X=$ $\mathbb{P}^{n} \times_{S} Y \rightarrow Y$. For any coherent $\Omega_{X}$-module $M$ we have the natural morphism

$$
\begin{aligned}
f_{*} D_{X}(M) \cong f_{*} R \mathcal{H o m}_{\Omega_{X}} & \left(M, \Omega_{X}\left[2 \operatorname{dim}_{S} X\right]\right) \\
& \rightarrow R \mathcal{H o m}_{\Omega_{Y}}\left(f_{*} M, f_{*} \Omega_{X}\left[2 \operatorname{dim}_{S} X\right]\right) \\
& \stackrel{\operatorname{Tr}_{p}}{\longrightarrow} R \mathcal{H o m}_{\Omega_{Y}}\left(f_{*} M, \Omega_{Y}\left[2 \operatorname{dim}_{S} Y\right]\right) \cong D_{Y}\left(f_{*} M\right) .
\end{aligned}
$$

It is enough to prove that this morphism is an isomorphism when $Y$ is affine, and $M \cong$ $q^{*} \mathcal{O}_{\mathbb{P}^{n}}(k)$, where $q: X \rightarrow \mathbb{P}^{n}$ is the second projection. This is straightforward.

Theorem 8.9 Suppose that $f: X \rightarrow Y$ is smooth and proper. Let $N \in \mathrm{D}^{\mathrm{co}}\left(\Omega_{X}\right.$-qcoh) and let $M \in \mathrm{D}^{\mathrm{abs}}\left(\Omega_{X}\right.$-coh). Then

$$
\left.R \mathcal{H} \operatorname{Hom}_{\Omega_{Y}}\left(f_{*} M, N\right)\right) \cong f_{*} R \mathcal{H o m}_{\Omega_{X}}\left(M, f^{!} N\right)
$$

Proof This follows from the projection formula, Proposition 8.4, and Theorem 8.8,

$$
\begin{aligned}
f_{*} R \mathcal{H o m}_{\Omega_{X}}\left(M, f^{!} N\right) & \cong f_{*}\left(D(M) \otimes_{\Omega_{X}}^{D} f^{!} N\right) \cong f_{*} D(M) \otimes_{\Omega_{Y}}^{D} N \\
& \cong D\left(f_{*} M\right) \otimes_{\Omega_{Y}}^{D} N \cong R \mathcal{H o m}_{\Omega_{Y}}\left(f_{*} M, N\right)
\end{aligned}
$$

The proof of the following result is the same as the proof of $[6,2.7 .1]$

Proposition 8.10 If $f: X \rightarrow Y$ is smooth, then

$$
D_{X} \circ f^{!} \cong f^{!} \circ D_{Y}[2(\operatorname{dim} X-\operatorname{dim} Y)] .
$$

Equivalently, $f^{+} \cong D_{X} \circ f^{!} \circ D_{Y}$.

\section{$9 \Omega$-modules and D-modules}

9.1 Positselski theorem Suppose that $S=\operatorname{Spec} k$, where $k$ is a filed of characteristic zero. Let $\mathcal{D}_{X}$ be the sheaf of differential operators on a smooth quasi-projective algebraic variety $X$ over $k$. We have an $\Omega_{X}-\mathcal{D}_{X}$-bimodule $\mathcal{D} \mathcal{R}_{X}=\Omega_{X} \otimes_{\mathcal{O}_{X}} \mathcal{D}_{X}$ with differential given in local coordinates $x_{1}, \ldots, x_{n}$ on $X$ by the formula

$$
d(\omega \otimes m)=d \omega \otimes m+\sum_{i=1}^{n} \omega \wedge d x_{i} \otimes \partial / \partial x_{i} m,
$$


where $m$ is a local section of $\mathcal{D}_{X}$ and $\omega$ is a local section of $\Omega_{X}$. The following result is an easy corollary of [11, B.2,B.5].

Theorem [11, B.2,B.5] The functor

$$
\mathrm{DR}_{X}=-\bigotimes_{\mathcal{D}_{X}} \mathcal{D R}_{X}[\operatorname{dim} X]: \mathcal{D}_{X} \text {-qcoh } \rightarrow \Omega_{X} \text {-qcoh }
$$

induces an equivalence of the unbounded derived category of quasi-coherent left $\mathcal{D}_{X}$-modules $\mathrm{D}\left(\mathcal{D}_{X}\right.$-qcoh) and the coderived category $\mathrm{D}^{\mathrm{co}}\left(\Omega_{X}\right.$-qcoh). Moreover, this functor induces an equivalence of the bounded derived category of coherent left $\mathcal{D}_{X^{-}}$ modules $\mathrm{D}^{b}\left(\mathcal{D}_{X^{-}}\right.$coh) and the absolute derived category $\mathrm{D}^{\mathrm{abs}}\left(\Omega_{X^{-}}\right.$coh $)$.

Lemma 9.2 Let $\mathcal{D R}_{X}^{-1}=\mathcal{D}_{X} \otimes \mathcal{O}_{X} \omega_{X}^{-1} \otimes \mathcal{O}_{X} \Omega_{X}$ be a $\mathcal{D}_{X}-\Omega_{X}$-bimodule with differential

$$
d\left(m \otimes \theta_{1} \wedge \cdots \wedge \theta_{r}\right)=\sum_{i=1}^{r} m \theta_{i} \otimes \theta_{1} \wedge \cdots \wedge \hat{\theta}_{i} \wedge \cdots \wedge \theta_{r}
$$

where $\theta_{1}, \ldots, \theta_{r}$ are commuting vector fields. Then there exists a natural coisomorphism of $\Omega_{X}$-modules $\mathcal{O}_{X} \rightarrow \mathcal{D} \mathcal{R}_{X}^{-1}[\operatorname{dim} X]$.

Proof The morphism is induced by the natural inclusion $\mathcal{O}_{X} \rightarrow \mathcal{D}_{X}$. The filtration of $\mathcal{D R}_{X}^{-1} / \mathcal{O}_{X}$ defined in $[6,1.5 .27]$ has acyclic associated graded complex. Each graded component is a bounded complex of $\mathcal{O}_{X}$-modules and thus coacyclic. The lemma follows from Lemma 3.2(c).

9.3 Let $f: X \rightarrow Y$ be a morphism of smooth quasi-projective algebraic varieties over $k$. Recall the definition of the direct image $f_{\star}$ and the extraordinary inverse image $f^{\dagger}$ for $\mathcal{D}$-modules [6]. The functor $f^{\dagger}$ is the derived functor of $f^{\bullet}(-) \otimes_{f} \bullet \mathcal{O}_{Y} \mathcal{O}_{X}$ shifted by $\operatorname{dim} X-\operatorname{dim} Y$. Let $\mathcal{D}_{Y \leftarrow X}=\omega_{X} \otimes_{f} \bullet \mathcal{O}_{Y} f^{\bullet}\left(\mathcal{D}_{Y} \otimes_{\mathcal{O}_{Y}} \omega_{Y}^{-1}\right)$ be a $\mathcal{D}_{X} f^{\bullet} \mathcal{D}_{Y^{-}}$ bimodule. Then $f_{\star}=R f_{\bullet}\left(\mathcal{D}_{Y \leftarrow X} \otimes_{\mathcal{D}_{X}}^{L}-\right)$. For coherent $\mathcal{D}_{X}$-modules we have the duality functor $\mathrm{D}_{X}^{\prime}(-)=R \mathcal{H}_{\mathcal{D}_{X}}\left(-, \mathcal{D}_{X} \otimes_{\mathcal{O}_{X}} \omega_{X}^{-1}\right)[\operatorname{dim} X]$. We are going to prove that the Positselski equivalence takes these functors to corresponding functors for $\Omega$-modules. We also prove that the Positselski equivalence is a tensor functor. By definition, the tensor product of left $\mathcal{D}_{X}$-modules $M$ and $N$ is the left $\mathcal{D}_{X}$-module $M \otimes \mathcal{O}_{X} N$ such that a vector field $\theta$ acts by the formula $\theta(m \otimes n)=\theta(m) \otimes n+$ $m \otimes \theta(n)$.

Theorem (a) There exists an equivalence of bifunctors from $\mathrm{D}\left(\mathcal{D}_{X}\right.$-qcoh $) \times$ $\mathrm{D}\left(\mathcal{D}_{X}\right.$-qcoh) to $\mathrm{D}^{\mathrm{co}}\left(\Omega_{X}-\mathrm{qcoh}\right)$ :

$$
\mathrm{DR}_{X}(-) \otimes_{\Omega_{X}}^{D} \mathrm{DR}_{X}(-) \cong \mathrm{DR}_{X}\left(-\otimes_{\mathcal{O}_{X}}^{L}-\right)
$$

(b) $f_{*}\left(\mathrm{DR}_{X}(-)\right) \cong \mathrm{DR}_{Y}\left(f_{\star}(-)\right)$.

(c) $f^{!}\left(\mathrm{DR}_{Y}(-)\right) \cong \mathrm{DR}_{X}\left(f^{\dagger}(-)\right)$.

(d) $\mathrm{D}_{X}\left(\mathrm{DR}_{X}(-)\right) \cong \mathrm{DR}_{X}\left(\mathrm{D}_{X}^{\prime}(-)\right)$. 
Proof (a) Let $M$ and $N$ be complexes of quasi-coherent $\mathcal{D}_{X}$-modules. Assume that $M$ is a complex of flat modules over $\mathcal{O}_{X}$. We have the following co-isomorphisms in $\mathrm{D}^{\mathrm{co}}\left(\Omega_{X}-\mathrm{qcoh}\right)$ :

$$
\begin{aligned}
\mathrm{DR}_{X}(M) \otimes_{\Omega_{X}}^{D} \mathrm{DR}_{X}(N) & \cong\left(\Omega_{X} \otimes_{\mathcal{O}_{X}} M\right) \otimes_{\Omega_{X}}\left(\Omega_{X} \otimes_{\mathcal{O}_{X}} N\right) \\
& \cong \Omega_{X} \otimes_{\mathcal{O}_{X}}\left(M \otimes_{\mathcal{O}_{X}} N\right) \cong \mathrm{DR}_{X}\left(M \otimes_{\mathcal{O}_{X}}^{D} N\right) .
\end{aligned}
$$

(b) Let $L$ be a complex of quasi-coherent flat $\mathcal{D}_{Y}$-modules. Then

$$
\begin{aligned}
f^{!}\left(\mathrm{DR}_{Y}(L)\right) & \cong \Omega_{X} \otimes_{f} \Omega_{Y} f^{\bullet}\left(\Omega_{Y} \otimes_{\mathcal{O}_{Y}} L\right)[2 \operatorname{dim} X-\operatorname{dim} Y] \\
& \cong \Omega_{X} \otimes_{f} \bullet \mathcal{O}_{Y} f^{\bullet} L[2 \operatorname{dim} X-\operatorname{dim} Y] \\
& \cong \Omega_{X} \otimes_{\mathcal{O}_{X}} f^{\dagger} L[\operatorname{dim} X] \cong \mathrm{DR}_{X}\left(f^{\dagger}(L)\right)
\end{aligned}
$$

(c) We give two proofs of this fact. First, the morphism $f$ is a composition of a closed embedding $i: X \rightarrow Z$ and a smooth projection $g: Z \rightarrow Y$. For example, $Z=X \times{ }_{S} Y$, and $i$ is the graph of $f$. Clearly, (c) follows from (b) and Theorems 4.5 and 4.7, since the adjoint functor is unique up to equivalence. Unfortunately, this equivalence depends on the choice of $g$ and $i$, and we give a more explicit second proof.

Let $M$ be a complex of flat quasi-coherent $\mathcal{D}_{X}$-modules. Then

$$
\begin{aligned}
& \operatorname{DR}_{Y}\left(f_{\star}(M)\right) \cong \Omega_{Y} \otimes_{\mathcal{O}_{Y}} R f_{\bullet}\left(\mathcal{D}_{Y \leftarrow X} \otimes_{\mathcal{D}_{X}} M\right)[\operatorname{dim} Y] \\
& \cong \Omega_{Y} \otimes_{\mathcal{O}_{Y}} R f_{\bullet}\left(f^{\bullet}\left(\omega_{Y}^{-1} \otimes_{\mathcal{O}_{Y}} \mathcal{D}_{Y}\right) \otimes_{f} \bullet \mathcal{O}_{Y} \omega_{X} \otimes_{\mathcal{D}_{X}} M\right)[\operatorname{dim} Y] \\
& \cong \Omega_{Y} \otimes_{\mathcal{O}_{Y}} R f_{\bullet}\left(f^{\bullet}\left(\omega_{Y}^{-1} \otimes_{\mathcal{O}_{Y}} \mathcal{D}_{Y}\right) \otimes_{f} \bullet \mathcal{O}_{Y} \Omega_{X} \otimes_{\mathcal{O}_{X}} \mathcal{D}_{X} \otimes_{\mathcal{D}_{X}} M\right)[\operatorname{dim} X+\operatorname{dim} Y] \\
& \cong \Omega_{Y} \otimes_{\mathcal{O}_{Y}} R f_{\bullet}\left(f^{\bullet}\left(\omega_{Y}^{-1} \otimes_{\mathcal{O}_{Y}} \mathcal{D}_{Y}\right) \otimes_{f} \bullet \mathcal{O}_{Y} \Omega_{X} \otimes_{\mathcal{O}_{X}} M\right)[\operatorname{dim} X+\operatorname{dim} Y] \text {, }
\end{aligned}
$$

and

$$
\begin{aligned}
& f_{*}\left(\mathrm{DR}_{X}(M)\right) \cong \Omega_{Y} \otimes_{\Omega_{Y}} f_{*}\left(\Omega_{X} \otimes_{\mathcal{O}_{X}} M\right)[\operatorname{dim} X] \\
& \quad \cong \Omega_{Y} \otimes_{\mathcal{O}_{Y}} \omega_{Y}^{-1} \otimes_{\mathcal{O}_{Y}} \mathcal{D}_{Y} \otimes_{\mathcal{O}_{Y}} \Omega_{Y} \otimes_{\Omega_{Y}} f_{*}\left(\Omega_{X} \otimes_{\mathcal{O}_{X}} M\right)[\operatorname{dim} X+\operatorname{dim} Y]
\end{aligned}
$$

where we used the quasi-isomorphism of right $\mathcal{D}_{X}$-modules $\Omega_{X} \otimes_{\mathcal{O}_{X}} \mathcal{D}_{X}[\operatorname{dim} X] \rightarrow$ $\omega_{X}$ (see $[6,1.5 .27]$ ), and the corresponding co-isomorphism of $\Omega_{Y}$-modules $\Omega_{Y} \otimes_{\mathcal{O}_{Y}} \omega_{Y}^{-1} \otimes_{\mathcal{O}_{Y}} \mathcal{D}_{Y} \otimes_{\mathcal{O}_{Y}} \Omega_{Y} \rightarrow \Omega_{Y}[\operatorname{dim} Y]$.

Choose an affine covering $\mathcal{U}$ of $X$. We can compute $f_{*}$ and $R f_{\bullet}$ using corresponding Čech resolutions. Thus it is enough to construct the equivalence when $X$ and $Y$ are affine. We have to check that the natural morphism

$$
\begin{aligned}
\Omega_{Y} \otimes_{\mathcal{O}_{Y}} \omega_{Y}^{-1} \otimes_{\mathcal{O}_{Y}} \mathcal{D}_{Y} \otimes \mathcal{O}_{Y} \Omega_{Y} \otimes_{\Omega_{Y}} \Omega_{X} \otimes_{\mathcal{O}_{X}} M \\
\rightarrow \Omega_{Y} \otimes_{\mathcal{O}_{Y}} \omega_{Y}^{-1} \otimes_{\mathcal{O}_{Y}} \mathcal{D}_{Y} \otimes_{\mathcal{O}_{Y}} \Omega_{X} \otimes_{\mathcal{O}_{X}} M
\end{aligned}
$$

commutes with differentials. This follows from definitions. 
(d) The functor $\mathrm{DR}_{X}^{-1}(-)=\mathcal{D R}_{X}^{-1} \otimes_{\Omega_{X}}-$ is quasi-inverse to $\mathrm{DR}_{X}$. Let $P$ be a perfect $\Omega_{X}$-module. We prove that $\mathrm{D}_{X}^{\prime}\left(\mathrm{DR}^{-1}(P)\right) \cong \mathrm{DR}_{X}^{-1}\left(\mathrm{D}_{X}(P)\right)$. Using the quasiisomorphism of left $\mathcal{D}_{X}$-modules $\mathcal{D}_{X} \otimes_{\mathcal{O}_{X}} \omega_{X}^{-1} \otimes_{\mathcal{O}_{X}} \Omega_{X}[\operatorname{dim} X] \rightarrow \mathcal{O}_{X}$ we get

$$
\begin{aligned}
& \mathrm{D}_{X}^{\prime}\left(\mathrm{DR}^{-1}(P)\right) \cong \mathcal{H o m}_{\mathcal{D}_{X}}\left(\mathcal{D}_{X} \otimes_{\mathcal{O}_{X}} \omega_{X}^{-1} \otimes_{\mathcal{O}_{X}} P, \mathcal{D}_{X} \otimes_{\mathcal{O}_{X}} \omega_{X}^{-1}\right)[\operatorname{dim} X] \\
& \cong \mathcal{H o m}_{\mathcal{D}_{X}}\left(\mathcal{D}_{X} \otimes_{\mathcal{O}_{X}} \omega_{X}^{-1} \otimes_{\mathcal{O}_{X}} P, \mathcal{D}_{X} \otimes_{\mathcal{O}_{X}} \omega_{X}^{-1} \otimes_{\mathcal{O}_{X}} \mathcal{O}_{X}[-\operatorname{dim} X]\right)[2 \operatorname{dim} X] \\
& \cong \mathcal{H o m}_{\mathcal{D}_{X}}\left(\mathcal{D}_{X} \otimes_{\mathcal{O}_{X}} \omega_{X}^{-1} \otimes_{\mathcal{O}_{X}} P,\right. \\
& \left.\quad \mathcal{D}_{X} \otimes_{\mathcal{O}_{X}} \omega_{X}^{-1} \otimes_{\mathcal{O}_{X}} \mathcal{D}_{X} \otimes_{\mathcal{O}_{X}} \omega_{X}^{-1} \otimes_{\mathcal{O}_{X}} \Omega_{X}\right)[2 \operatorname{dim} X] .
\end{aligned}
$$

We have the natural transformation

$$
\begin{aligned}
\Phi: & \mathrm{DR}_{X}^{-1}\left(\mathrm{D}_{X}(P)\right)[-2 \operatorname{dim} X] \cong \mathcal{D}_{X} \otimes \mathcal{O}_{X} \omega_{X}^{-1} \otimes \mathcal{O}_{X} \mathcal{H o m}_{\Omega_{X}}\left(P, \Omega_{X}\right) \\
& \rightarrow \mathcal{H o m}_{\mathcal{D}_{X}}\left(\mathcal{D}_{X} \otimes_{\mathcal{O}_{X}} \omega_{X}^{-1} \otimes \mathcal{O}_{X} P, \mathcal{D}_{X} \otimes_{\mathcal{O}_{X}} \omega_{X}^{-1} \otimes_{\mathcal{O}_{X}} \mathcal{D}_{X} \otimes_{\mathcal{O}_{X}} \omega_{X}^{-1} \otimes_{\mathcal{O}_{X}} \Omega_{X}\right)
\end{aligned}
$$

given over the affine subscheme $U \subset X$ by the formula $m \otimes \varphi \mapsto(d \otimes p \mapsto$ $(m \otimes d \otimes \varphi(p))$, where $\varphi \in \mathcal{H}^{\prime} m_{\Omega_{X}}\left(P, \Omega_{X}\right)(U), p \in P(U), m, d \in \mathcal{D}_{U} \otimes_{\mathcal{O}_{U}} \omega_{U}^{-1}$. We claim that the transformation is an isomorphism of $\mathcal{D}_{X}$-modules. As usual, it is enough to prove the claim locally and for $P=\mathcal{O}_{X}$. This is clear.

Remark The internal Hom for $\Omega_{X}$-modules corresponds to the internal Hom for $\mathcal{D}$ modules under Positselski equivalence. The last bifunctor is defined as follows:

$$
R \operatorname{Hom}_{\mathcal{D}_{X}}(M, N)=\mathrm{D}_{X}^{\prime}(M) \otimes_{\mathcal{O}_{X}}^{L} N
$$

where $M$ is coherent, and $N$ is quasi-coherent.

Acknowledgments The author is very grateful to Leonid Positselski for explaining his results on exotic derived categories and for many useful conversations. He also thanks Sergey Arkhipov and Sergey Gorchinskiy for helpful discussions on the topic.

\section{References}

1. Beilinson, A.A., Bernstein, J., Deligne, P.: Faisceaux pervers. In: Analysis and Topology on Singular Spaces, vol. I (Luminy, 1981), Astérisque, 100, pp. 5-171. Société Mathématique de France, Paris (1982)

2. Beilinson, A., Drinfeld, V.: Quantization of Hitchin's Integrable System and Hecke Eigenvalues. http:// www.math.uchicago.edu/ mitya/langlands/hitchin/BD-hitchin

3. Bondal, A.I., Kapranov, M.M.: Framed triangulated categories. Math. USSR-Sb. 70(1), 93-107 (1991)

4. Eisenbud, D.: Commutative Algebra. Graduate Texts in Mathematics, vol. 150. Springer, New York (1995)

5. Hartshorne, R.: Residues and Duality. Lecture Notes in Mathematics, vol. 20. Springer, Berlin (1966)

6. Hotta, R., Takeuchi, K., Tanisaki, T.: D-Modules, Perverse Sheaves, and Representation Theory. Progress in Mathematics, vol. 236. Birkhaüser, Boston (2008)

7. Kapranov, M.M.: On DG-modules over the de Rham complex and the vanishing cycles functor. In: Algebraic Geometry (Chicago, 1989), Lecture Notes in Mathematics, vol. 1479, pp. 57-86. Springer, Berlin (1991) 
8. Neeman, A.: The connection between the $K$-theory localization theorem of Thomason, Trobaugh and Yao and the smashing subcategories of Bousfield and Ravenel. Ann. Sci. École Norm. Sup. (4) 25(5), 547-566 (1992)

9. Polishchuk, A., Positselski, L.: Hochschild (co)homology of the second kind I. arXiv:1010.0982

10. Positselski, L.: Homological Algebra of Semimodules and Semicontramodules. Monografie Matematyczne, vol. 70. Birkhäuser, Basel (2010)

11. Positselski, L.: Two Kinds of Derived Categories, Koszul Duality, and Comodule-Contramodule Correspondence. Memoirs of the American Mathematical Society, vol. 212(996). American Mathematical Society, Providence (2011)

12. Positselski, L.: Positselski's LiveJournal. http://posic.livejournal.com/tag/math4 (in Russian)

13. Positselski, L.: Coherent analogues of matrix factorizations and relative singularity categories. arXiv:1102.0261 